\title{
No-Arbitrage, State Prices and Trade in Thin Financial Markets *
}

\author{
Andrés Carvajal ${ }^{\dagger} \quad$ Marek Weretka ${ }^{\ddagger}$
}

December 16, 2008

\begin{abstract}
We examine how non-competitiveness in financial markets affects the choice of asset portfolios and the determination of equilibrium prices. We apply a model of economic equilibrium, based on [12], in which individual traders recognize and estimate the impact of their trades on financial prices, and in which these effects are determined endogenously as part of the equilibrium concept. For the case in which markets allow for perfect insurance, we argue that the principle of no-arbitrage asset pricing is consistent with non-competitive behavior and extend the fundamental theorem of asset pricing to the non-competitive setting.
\end{abstract}

Key words: asset pricing; institutional traders; price impact; thin markets; pricing kernel.

JEL classification numbers: D43, D53, G11, G12, L13

The model of competitive equilibrium imposes the premise that all agents intervening in the markets observe a price that they take as given: they assume that their trade in the market, however large, can be made at that fixed price. In finance, the theory of the determination of the prices of financial derivatives, and the formulæ derived from this theory and applied in practice, are based on the premise that asset prices should permit no costless and riskless opportunities for profit; under this condition, asset prices embed an "objective" probability distribution that can be used to obtain the price of any asset as its discounted expected return: this is the basic content of the Fundamental Theorem

* A. Carvajal acknowledges the financial support of a Warwick-RSS grant, and the hospitality of the Department of Economics at the University of Wisconsin at Madison.

$\dagger$ CRETA and Department of Economics, University of Warwick, Coventry CV4 7AL, United Kingdom; Phone: +44-(0)2476-523040; Fax: +44-(0)2476-523032; e-Mail address: A.M.Carvajal@Warwick.ac.uk

$\ddagger$ University of Wisconsin-Madison, Department of Economics, 1180 Observatory Drive, Madison, WI 53706. Phone: (608) 262 2265; e-Mail address: mweretka@ssc.wisc.edu. 
of Asset Pricing, of its applications (the Modigliani-Miller theorem, for instance), and of the formulae developed to price financial derivatives (for example, the Black-Scholes formula). When the competitive model is applied in a setting of uncertainty and trade of financial assets, the premise of finance holds at equilibrium: under minimal assumptions, at competitive equilibrium prices there should be no arbitrage opportunities, and hence the theory of asset pricing is immediately applicable.

The assumption that all agents ignore the impact of their trades on prices, however, appears at odds with empirical evidence from crucial markets. In financial markets, even as deep as NYSE or NASDAQ, the evidence is clear in this direction: large institutional traders have non-negligible price impacts, which they do take into account when designing their trading strategies, and tools have been developed to implement portfolio recompositions taking into account their price impacts. ${ }^{1}$

Attempts to develop a general equilibrium framework in which traders do take into account their price impacts have so far proved to be less successful. The work initiated by T. Negishi in [8], though interesting, either required the imposition of very strong assumptions, or gave highly complex, intractable and inapplicable models: in the Negishi's approach, traders were allowed to have subjective perceptions of the price impact, which implied a great deal of arbitrariness on the determination of equilibria, unless the subjective conjectures where exogenously restricted; later attempts to impose more structure to the definition of equilibrium endowed the model with explicit strategic games, which came at the cost of tractability. ${ }^{2}$

In this paper, we apply a model of economic equilibrium, based on [12], in which all individual traders recognize the impact that their trades have on financial prices, and in which these effects are determined endogenously as part of the equilibrium concept. ${ }^{3}$ This model maintains the flexible, tractable and spare structure of the competitive model, but the departure from the competitive premise implies that none of the standard positive or normative results are immediately applicable, and, in particular, the necessity of a noarbitrage condition, and the derivation of an objective probability have to be argued. We prove that, even in the absence of competitive behavior, the principle of no-arbitrage is consistent with equilibrium pricing in financial markets, and we extend the Fundamental Theorem of Asset Pricing to the noncompetitive setting; to the best of our knowledge, there is no previous study of noncompetitive markets with many arbitrary assets that possibly have collinear payoffs. ${ }^{4}$ In this paper, we concentrate on the case in which the

\footnotetext{
${ }^{1}$ For literature on this, see [6], [2] and [9]. Importantly, these large traders constitute a very significant portion of volume of trade in financial markets: see [3].

2 For a review of this literature, see [5].

3 This is the feature that closes the definition of equilibrium imposing interesting structure.

4 In the important literature on financial microstructure, price impacts of the traders result from asymmetric information, rather than from the thinness of the markets - see, for instance, the work of Kyle [7] and Vayanos [10]. We believe that our work is complementary to this literature.
} 
existing financial markets allow for complete insurance against risk.

This paper is part of a research program in which we study the properties of abstract thin markets. As a part of this program, [13] shows that the concept of equilibrium from this paper refines a Subgame Perfect Nash equilibrium in the game defined by the Walrasian auction. There, it is also argued that the equilibrium outcome will be observed in anonymous markets, in which investors have no other information but their past trades and market prices, and therefore they discover market power through a statistical inference by estimating slopes of their demands using the Least Squares method. Therefore the equilibrium has a game theoretic foundation endowed with the learning process by which anonymous markets converge to this equilibrium. We believe that the assumption of anonymity is approximately satisfied in financial markets, in which large institutional investors know that they have price impacts, but very often have no information about those with whom they are trading.

\section{The ECONOMY}

Consider a financial market that evolves over two periods. Suppose that in the second period there is a finite number of states of the world, indexed by $s=1, \ldots, S$, while in the first period investors trade assets that deliver a return in the second period. There is only one good, the numèraire, and all assets are denominated in this commodity. There is a finite number of assets, indexed by $a=1, \ldots, A$, and their payoffs are uncertain: in the second period, asset $a$ pays $R_{s}^{a}$ in state $s$. Vector $R^{a}=\left(R_{1}^{a}, \ldots, R_{S}^{a}\right)^{\top}$ is a commonlyknown random variable, and matrix $R=\left(R^{1}, \ldots, R^{A}\right)$, which is of dimensions $S \times A$, denotes the financial structure of the economy. ${ }^{5}$ We restrict our attention to complete financial markets: we assume that there are at least as many assets with linearly independent payoffs as states of the world. Then, without further loss of generality, we assume that the first $S$ assets are linearly independent: we partition $R=\left(R_{S}, R_{A-S}\right)$, and assume that the $S \times S$ matrix $R_{S}$ is nonsingular.

There is a finite number of investors, who trade assets among themselves; a typical investor is indexed by $i=1, \ldots, I$. Investor $i$ is endowed with a future wealth of $e_{s}^{i}>0$, contingent on the state of the world, and we denote by $e^{i}=\left(e_{1}^{i}, \ldots, e_{S}^{i}\right)$ the random variable representing investor $i$ 's future endowment of wealth. Each trader has von Neumann-Morgenstern preferences with respect to her wealth (or consumption) in the second period, with cardinal utility indices $u_{s}^{i}: \mathbb{R}_{++} \rightarrow \mathbb{R}$. We assume that each utility index is twice continuously differentiable, differentiably strictly monotonic and differentiably strictly concave, and satisfies Inada conditions. For notational simplicity,

\footnotetext{
${ }^{5}$ If we denote by $\mathbb{I}_{n}$ the $n \times n$ identity matrix, then $R=\mathbb{I}_{S}$ is the case of a full set of elementary securities.
} 
henceforth we write $u^{i}\left(\left(c_{s}^{i}\right)_{s=1}^{S}\right)=\sum_{s} u_{s}^{i}\left(c_{s}^{i}\right)$; by our assumptions, $\partial u^{i} \gg 0,{ }^{6}$ while $\partial^{2} u^{i}$ is a diagonal, negative definite matrix.

The economy is completely characterized by the triple of a profile of individual preferences, a profile of future endowments of wealth and an asset payoff structure, namely $\{u, e, R\}=\left\{\left(u^{i}\right)_{i=1}^{I},\left(e^{i}\right)_{i=1}^{I}, R\right\}$.

\section{Equilibrium in Thin Markets}

As there is only one commodity, trade takes place only in the market for assets in the first period. Asset prices are denoted by $P=\left(P_{1}, \ldots, P_{A}\right)^{\top}$, and the portfolio of trader $i$ is denoted by $\Theta^{i}{ }^{7}$ For each investor $i$, let $M_{a, a^{\prime}}^{i}$ measure individual her belief of how the price of asset $a$ changes when she expands her buy order for asset $a^{\prime}$ by one share. In the competitive model, this measure is exogenously fixed at zero; here, we follow [12] in letting $M_{a, a^{\prime}}^{i}$ be endogenously determined as part of the equilibrium concept. The $A \times A$ matrix

$$
M^{i}=\left(\begin{array}{ccc}
M_{1,1}^{i} & \ldots & M_{1, A}^{i} \\
\vdots & \ddots & \vdots \\
M_{A, 1}^{i} & \ldots & M_{A, A}^{i}
\end{array}\right)
$$

will be referred to as trader $i$ 's price impact matrix: she believes that the price change exerted by a perturbation $\Delta$ to her portfolio is $M^{i} \Delta$. We will restrict attention only to positive definite and symmetric price impact matrices, and it will be a part of the definition of equilibrium that the profile of individual matrices is mutually consistent, in the sense that each matrix $M^{i}$ correctly measures the price impacts of trader $i$, given the individual price impacts perceived by all other traders in the market.

Intuitively, an equilibrium in thin financial markets will be a triple consisting of a vector of asset prices, a profile of portfolios and a profile of price impact matrices, $(\bar{P}, \bar{\Theta}, \bar{M})$, such that:

1. all markets clear;

2. all traded portfolios are individually optimal, given the price impacts perceived by agents: for each trader $i$, at prices $\bar{P}$, trade $\bar{\Theta}^{i}$ is optimal, given that any other trade, $\tilde{\Theta}^{i}$, would change prices to $\bar{P}+\bar{M}^{i}\left(\tilde{\Theta}^{i}-\bar{\Theta}^{i}\right)$; and

3. all perceived price impact matrices correctly estimate the effects of individual portfolio perturbations on the prices that would be required for the rest of the market to optimally absorb them: for each trader $i$, (small) perturbations to her portfolio, $\Delta^{i}$, uniquely define a price at which the other traders of the market, given their price impacts, are willing to supply $\Delta^{i}$ to $i$, and the derivative of this mapping at equilibrium trade $\bar{\Theta}^{i}$ is $\bar{M}^{i}$.

${ }^{6}$ We take this vector as a column.

7 This means that the future numèraire wealth of investor $i$ is given by $e^{i}+R \Theta^{i}$, while the cost of her portfolio, if she constitutes it at prices $\tilde{P}$, is $\tilde{P} \cdot \Theta^{i}$, which she incurs in the first period. 
The first condition in the definition is standard, as in the definition of competitive equilibrium; the other two are not, so we now proceed to their formal presentation.

\subsection{Stable Individual Trade}

Here, each trader perceives that her trade affects prices, and takes these impacts into account when designing her investment strategy. We assume that trader $i$ wants to maximize the ex-ante utility of her future wealth, net of the present cost of constituting her portfolio. ${ }^{8}$ Critically, when computing the cost of constituting her portfolio, trader $i$ will take into account (her perception of) the effect that her portfolio has on prices. To avoid confusion with the competitive model, we refer to this condition as stability.

Definition 1. Given prices $P$ and a price impact matrix $M^{i}$, portfolio $\bar{\Theta}^{i}$ is stable for individual $i$ if it solves program

$$
\max _{\Theta^{i}}-\left(P+M^{i}\left(\Theta^{i}-\bar{\Theta}^{i}\right)\right) \cdot \Theta^{i}+u^{i}\left(e^{i}+R \Theta^{i}\right) .
$$

The second condition in the definition of equilibrium will be the requirement that, given their beliefs on price impacts, no trader will find that she can increase her (net) ex-ante utility by demanding a different portfolio: trader $i$ 's portfolio should be stable given equilibrium prices and her perceived price impacts at equilibrium trade. ${ }^{9}$ It requires that for each trader, given her price impact, no deviation from the equilibrium trade be able improve her ex-ante utility.

Given the assumption of strong concavity of preferences and the condition that price impact matrices are to be positive definite, stability of portfolio $\bar{\Theta}^{i}$, given $\left(P, M^{i}\right)$, is characterized by the condition that

$$
P+M^{i} \bar{\Theta}^{i}=R^{\top} \partial u^{i}\left(e^{i}+R \bar{\Theta}^{i}\right)
$$

\subsection{Subequilibrium and Consistent Price Impact Matrices}

The third condition of the definition of equilibrium requires the perceived price impacts of all traders to be accurate estimates of their actual price impacts, at least locally and to a

8 Formally, we are assuming that individual preferences are quasilinear on the wealth of the first period. We will not impose non-negativity constraints on this variable, hence we do not need to specify individual endowments of present wealth.

9 Suppose that trader $i$ is faced with the inverse demand function

$$
p(\Theta)=P+M^{i}(\Theta-\bar{\Theta}) \cdot \Theta
$$

from the rest of the market, where $\bar{\Theta}$ is some reference point. For each $\bar{\Theta}$, let

$$
\Gamma^{i}(\bar{\Theta})=\operatorname{argmax}_{\Theta}-\left(P+M^{i}(\Theta-\bar{\Theta})\right) \cdot \Theta+u^{i}\left(e^{i}+R \Theta\right) .
$$

The stability condition requires that $\bar{\Theta}^{i}$ be a fixed point of this mapping. 
first order, given the perceived price impacts of every other trader. Unlike in a competitive market, each trader here knows that prices must endogenously adjust to encourage other traders to (optimally) accommodate her trade. Consequently, in this model markets clear and other investors respond optimally to prices, even if one of the investors is trading a suboptimal portfolio; this feature is captured by the notion of subequilibrium in financial markets, defined below.

DEFINITION 2. Given an individual $i$ and a profile of price impact matrices for all traders but $i, M^{-i}=\left(M^{j}\right)_{j \neq i}$, a subequilibrium triggered by trade $\tilde{\Theta}^{i}$ is a pair of a vector prices and a profile of trades by all other investors, $\left(\tilde{P}, \tilde{\Theta}^{-i}\right)$, such that:

1. all markets clear: $\tilde{\Theta}^{i}+\sum_{j \neq i} \tilde{\Theta}^{j}=0$;

2. all traders other than $i$ act optimally: for each $j \neq i$, trade $\tilde{\Theta}^{j}$ is stable given $\left(\tilde{P}, M^{j}\right)$.

For each $i$, let $\mathcal{S}^{i}\left(\tilde{\Theta}^{i} ; M^{-i}\right)$ be the set of all subequilibria triggered by trade $\tilde{\Theta}^{i}$, given that the other traders in the market perceive their price impacts as $M^{-i}$, and let $\mathcal{P}^{i}\left(\tilde{\Theta}^{i} ; M^{-i}\right)$ be the projection of that set into the space of prices. If $\mathcal{P}^{i}\left(\tilde{\Theta}^{i} ; M^{-i}\right)$ is singleton-valued in a neighborhood of some $\bar{\Theta}^{i}$, it defines, at least locally, an inverse demand function, $P^{i}\left(\tilde{\Theta}^{i}\right)$, faced by trader $i$ given $M^{-i}$. If such function is differentiable, trader $i$ can estimate its derivatives. The condition of consistency of price impact matrices is that, at equilibrium, perceived price impacts should not be arbitrary: each trader is able to estimate the slope of her real inverse demand, so that the inverse demand function implicit in the condition of stability of her trade is a correct first-order approximation to her real inverse demand, given the estimations made by all other traders. ${ }^{10}$ This concept of mutual consistency of price impact estimations, which completes the definition of equilibrium, is formalized next.

Definition 3. A profile $\bar{M}$ of price impact matrices (for all traders) is mutually consistent given prices and trades $(\bar{P}, \bar{\Theta})$ if, for each individual $i$, there exist open neighborhoods $N^{i}\left(\bar{\Theta}^{i}\right)$ and $N^{i}(\bar{P})$, and a differentiable function $P^{i}: N^{i}\left(\bar{\Theta}^{i}\right) \rightarrow N^{i}(\bar{P})$, such that:

1. locally, $P^{i}$ is the inverse demand faced by $i$ :

$$
\mathcal{P}^{i}\left(\tilde{\Theta}^{i} ; M^{-i}\right) \cap N^{i}(\bar{P})=\left\{P^{i}\left(\tilde{\Theta}^{i}\right)\right\}
$$

for every $\tilde{\Theta}^{i} \in N^{i}\left(\bar{\Theta}^{i}\right)$; and

2. matrix $\bar{M}^{i}$ is a correct estimation of the Jacobean of $P^{i}$ at $\bar{\Theta}^{i}: \partial P^{i}\left(\bar{\Theta}^{i}\right)=\bar{M}^{i}$.

10 The approximation assures that in the definition of stability the second order condition is always satisfied. Otherwise, the approximation is without loss of generality. 
Note that consistency is imposed on the whole profile of individual matrices, and not matrix by matrix: the second and third conditions of the definition require that $\partial_{\tilde{\Theta}^{i}} P^{i}\left(\bar{\Theta}^{i} ; M^{-i}\right)=M^{i}$ for every trader. ${ }^{11}$ Intuitively, consistent price impact matrices reflect the price changes needed to clear the market for any small deviation $\tilde{\Theta}^{i}$ by trader $i$, given that other traders respond rationally to market prices in any subequilibrium. This means that each trader correctly estimates her price impact, to a first order, and incorporates this information into her individual decision problem. This contrasts with the competitive model, in which traders incorrectly assume that their price impacts are null; put simply, traders in thin markets do not act as price takers, and behave as "slope takers" instead.

\subsection{Equilibrium}

The three principles of equilibrium in thin financial markets have now been introduced: equilibrium is a triple of prices, trades and perceptions of price impacts such that all markets clear; all traders are acting optimally, given the perception they hold of their price impacts; and these perceptions are accurate estimates of the actual price impacts exerted by the traders.

DEFINITION 4. An equilibrium in thin financial markets for economy $\{u, e, R\}$ is a triple consisting of a vector of asset prices, a profile of portfolios and a profile of price impact matrices, $(\bar{P}, \bar{\Theta}, \bar{M})$, such that:

1. $\sum_{i} \bar{\Theta}^{i}=0$;

2. for each trader $i$, trade $\bar{\Theta}^{i}$ is stable given $\left(\bar{P}, \bar{M}^{i}\right)$; and

3. the profile of price impact matrices $\bar{M}$ is mutually consistent, given prices and trades $(\bar{P}, \bar{\Theta})$.

Importantly, note that at equilibrium all trades take place at prices $\bar{P}$. The following lemma provides a characterization of equilibrium for the case when there are no redundant assets in the market. For simplicity of notation, given an array of $I-1$ positive definite matrices, $\Delta$, let $\mathcal{H}(\Delta)$ denote the harmonic average of the array. ${ }^{12}$

Lemma 1. Suppose that $R$ is nonsingular. ${ }^{13}$ Then, $(\bar{P}, \bar{\Theta}, \bar{M})$ is an equilibrium in thin financial markets for economy $\{u, e, A\}$ if, and only if,

\footnotetext{
${ }^{11}$ Strictly speaking, the intuition given before the definition is slightly weaker than the definition itself: we do not require that subequilibrium prices be unique (i.e., that $\mathcal{P}^{i}\left(\tilde{\Theta}^{i} ; M^{-i}\right)$ be singleton-valued), but only that they be determinate, or locally unique.

12 That is, $\mathcal{H}\left(\left(\Delta^{j}\right)_{j \in \mathcal{J}}\right)=(I-1)\left(\sum_{j \in \mathcal{J}}\left(\Delta^{j}\right)^{-1}\right)^{-1}$.

13 So that $A=S$ and $R_{S}=R$.
} 
1. $\sum_{i} \bar{\Theta}^{i}=0$;

2. for each trader $i, P+M^{i} \bar{\Theta}^{i}=R^{\top} \partial u^{i}\left(e^{i}+R \bar{\Theta}^{i}\right)$;

3. for each trader $i$,

$$
M^{i}=\frac{1}{I-1} \mathcal{H}\left(\left(M^{j}-R^{\top} \partial^{2} u^{j}\left(e^{j}+R \bar{\Theta}^{j}\right) R\right)_{j \neq i}\right) .
$$

Proof: See Appendix A1.

Q.E.D.

\section{Arbitrage and Irrelevant Price Impacts}

The primary objective of this paper is to demonstrate that equilibrium in thin financial markets is not inconsistent with the standard no-arbitrage principle. Therefore, we now review the concept of no-arbitrage, introduce a weaker version of that principle, and also introduce an analogous condition, which we call the "no irrelevant impact" condition, for price impact matrices, and discuss its implication for economies with elementary securities.

\subsection{No-Arbitrage}

A vector of asset prices $P$ allows no arbitrage opportunities if $R \Theta>0$ implies $P \cdot \Theta>0$. It is well known that price vector $P$ allows no-arbitrage opportunities if, and only if, there exists a vector of strictly positive "state prices," $p \in \mathbb{R}_{++}^{S}$, that determines the value of income in each state of the world: $P=R^{\top} p$. Consequently, the set of asset prices that allow no arbitrage opportunities is the convex, open, positive cone

$$
\left\{P \in \mathbb{R}^{A} \mid \exists p \in \mathbb{R}_{++}^{S}: P=R^{\top} p\right\}
$$

A weaker condition of no-arbitrage would be the requirement that portfolios whose future return is zero in all states of the world should have zero cost at market prices. That is, we will say that a vector of asset prices $P$ allows no weak arbitrage opportunities if $R \Theta=0$ implies $P \cdot \Theta=0$. This weaker no-arbitrage condition amounts to a relaxation of the requirement that state prices be strictly positive, as in the following lemma.

LEMMA 2. The set of asset prices that allow no weak arbitrage opportunities is

$$
\left\{P \in \mathbb{R}^{A} \mid \exists p \in \mathbb{R}^{S}: P=R^{\top} p\right\}
$$

Proof: See Appendix A1.

Q.E.D.

If a trader who has monotone preferences and takes prices as given can find an optimal portfolio, it must be that the prices of the assets allow neither strong nor weak arbitrage 
opportunities; in particular, in a competitive equilibrium of the economy we are considering there can be no arbitrage opportunities. In the following sections we show that when markets are thin, only the weak notion of no-arbitrage is implied by optimization of a rational agent. ${ }^{14}$ In order to claim the strong no-arbitrage condition, one also needs to invoke the market clearing condition.

Henceforth, we say that a pair of vectors of prices $(P, p) \in \mathbb{R}^{A} \times \mathbb{R}^{S}$ is $R$-associated if they satisfy $P=R^{\top} p$. By our assumption that markets are complete, it is immediate that $(P, p)$ is $R$ - associated only if $p=\left(R_{S}^{\top}\right)^{-1}\left(P_{1}, \ldots, P_{S}\right)^{\top}$. Consequently, $R$-association of prices defines a bijection between the linear subspace of all asset prices $P$ satisfying the weak no-arbitrage condition, set $(9)$, and $\mathbb{R}^{S}$. In the case of strong arbitrage, such bijection is between a convex cone, set $(8)$, and $\mathbb{R}_{++}^{S}$.

If $(P, p)$ is $R$-associated and $P$ allows no arbitrage opportunities in the strong sense, then vector $\left(\sum_{s} p_{s}\right)^{-1} p \gg 0$ is a probability measure often referred to as the pricing kernel implied by $P$. Similarly, if $P$ allows no weak arbitrage opportunities, then vector $p$ can be interpreted as a (weak) pricing kernel implied by $P$. Note, however, that the weak pricing kernel does not have to be a probability measure since prices do not necessarily sum up to one and for some states they can even take negative values.

\subsection{PRICE IMPACT}

A condition on price impact matrices that is analogous to the no-arbitrage principle on asset prices is the requirement that only portfolios with non-zero future payoff should affect prices. That is, we say that a price impact matrix $M^{i}$ gives no irrelevant impact if $R \Theta^{i}=0$ implies $M^{i} \Theta^{i}=0$. Technically, $M^{i}$ gives no irrelevant impact if the kernel of matrix $M^{i}$ contains the kernel of matrix $R$. It is immediate that, under this condition, portfolios with identical payoffs should have identical price effects. The next result provides a unique decomposition for symmetric matrices that give no irrelevant price impact; for any $M^{i}$ define $m^{i}=\left(R_{S}^{\mathrm{T}}\right)^{-1} M_{S}^{i}\left(R_{S}\right)^{-1}$, where $M_{S}^{i}$ is the $S \times S$ leading principal minor of matrix $M^{i}$.

Lemma 3. Let $M^{i}$ be an $A \times A$, symmetric price impact matrix. If $M^{i}$ gives no irrelevant impact, then

1. $M^{i}$ can be written as $M^{i}=R^{\top} m^{i} R$; and

2. $m^{i}$ is the only matrix that allows for such decomposition of $M^{i}$.

Proof: See Appendix A1.

Q.E.D.

14 For this result, one also needs to assume the condition of no irrelevant impact, defined in the following section. 
In the same vein as with prices, we say that a pair of an $A \times A$ matrix and an $S \times S$ matrix $\left(M^{i}, m^{i}\right)$ is $R$-associated if $M^{i}=R^{\top} m^{i} R$. From Lemma 3, it follows that if $M^{i}$ is symmetric and gives no irrelevant impact, then $\left(M^{i}, m^{i}\right)$ is $R$-associated if, and only if, $m^{i}=\left(R_{S}^{\top}\right)^{-1} M_{S}^{i}\left(R_{S}\right)^{-1}$. Consequently, $R$-association defines a bijection between these sets of $A \times A$ and $S \times S$ matrices. The next two results show that the presence of one investor who trades optimally and whose trade has no irrelevant impact is sufficient for the weak no-arbitrage condition.

Proposition 1. Suppose that price impact matrix $\bar{M}^{i}$ gives no irrelevant impact, and that there exists a portfolio $\bar{\Theta}^{i}$ that is stable for $i$ given $\left(\bar{P}, \bar{M}^{i}\right)$. Then, prices $\bar{P}$ allow no weak arbitrage opportunities.

Proof: By stability of the individual's portfolio, it follows that

$$
\bar{P}+\bar{M}^{i} \bar{\Theta}^{i}=R^{\top} \partial u^{i}\left(e^{i}+R \bar{\Theta}^{i}\right)
$$

Multiplying (10) by any $\Theta^{i}$ such that $R \Theta^{i}=0$ gives

$$
\bar{P} \cdot \Theta^{i}=\partial u^{i}\left(e^{i}+R \bar{\Theta}^{i}\right)^{\top} R \Theta^{i}-\left(\bar{\Theta}^{i}\right)^{\top} \bar{M}^{i} \Theta=0,
$$

since $\bar{M}^{i}$ gives no irrelevant price impact.

Q.E.D.

Intuitively, changes in the portfolio that do not affect payoffs do not alter consumption either, and, hence, do not change the marginal rate of substitution between assets. Since, at an optimum, the marginal rate of substitution equals marginal revenue, $\bar{P}+\bar{M}^{i} \bar{\Theta}^{i}$, the latter is not affected either. But, with no irrelevant impact, marginal revenue is constant only if prices are orthogonal to zero-payoff portfolios.

Corollary 1. If $(\bar{P}, \bar{\Theta}, \bar{M})$ is an equilibrium in thin financial markets for $\{u, e, R\}$, and if for at least one trader her price impact matrix gives no irrelevant impact, then $\bar{P}$ allows no weak arbitrage opportunities, and there exists a (weak) pricing kernel, $p \in \mathbb{R}^{S}$ such that $P=R^{\top} p$.

Proof: This follows from Proposition 1 and Lemma 2.

Q.E.D.

The next result now demonstrates that, in equilibrium, if all traders but one estimate that they have no irrelevant price impact, then that remaining trader will automatically have no irrelevant impact.

Proposition 2. Let $(\bar{P}, \bar{\Theta}, \bar{M})$ be an equilibrium in thin financial markets for economy $\{u, e, R\}$. If for all traders $j \neq i$ their price impact matrices give no irrelevant price impact, then matrix $\bar{M}^{i}$ gives no irrelevant impact either. 
Before the proof of the proposition, we introduce a lemma whose proof we again defer to Appendix A1.

LEMMA 4. Return-equivalent deviations trigger the same subequilibrium prices: Fix an individual $i$ and a profile of price impact matrices for all traders but $i, \bar{M}^{-i}=\left(\bar{M}^{j}\right)_{j \neq i}$, and suppose that each matrix $\bar{M}^{j}$ gives no irrelevant price impact. Suppose that portfolios $\tilde{\Theta}^{i}$ and $\hat{\Theta}^{i}$ are such that $R \tilde{\Theta}^{i}=R \hat{\Theta}^{i}$. If $\left(\tilde{P}, \tilde{\Theta}^{-i}\right)$ is a subequilibrium triggered by trade $\tilde{\Theta}^{i}$, then

$$
\left(\tilde{P},\left(\tilde{\Theta}^{j}+(I-1)^{-1}\left(\tilde{\Theta}^{i}-\hat{\Theta}^{i}\right)\right)_{j \neq i}\right)
$$

is a subequilibrium triggered by trade $\hat{\Theta}^{i}$.

Proof of Proposition 2: Let $\Theta^{i}$ be a portfolio such that $R \Theta^{i}=0$. By definition of equilibrium, there exists a local inverse demand function for individual $i, P^{i}$. Define the function $\varphi(\lambda)=P^{i}\left(\bar{\Theta}^{i}+\lambda \Theta^{i}\right)$, for real numbers $\lambda$ close enough to 0. By Lemma 4, it follows that $\left(\bar{P},\left(\bar{\Theta}^{j}-(I-1)^{-1} \lambda \Theta^{i}\right)_{j \neq i}\right)$ is a subequilibrium triggered by trade $\lambda \Theta^{i}$, so it is immediate that $\varphi^{\prime}(\lambda)=0$. By construction, $\varphi^{\prime}(0)=\partial P^{i}\left(\bar{\Theta}^{i}\right) \Theta^{i}=\bar{M}^{i} \Theta^{i}$. Q.E.D.

We restrict our attention to equilibria in which all price impact matrices give no irrelevant impact. In the following sections we argue that such equilibria exist and are determinate. We conjecture that equilibria in which traders have irrelevant impact do not exist.

\section{Pricing Kernel in Thin Complete Markets}

\subsection{Elementary Securities}

Since we are considering only the case of complete financial markets, we can obtain a canonical auxiliary representation of the economy by endowing with a complete set of elementary securities, in lieu of the set of securities $R$. That is, the representation of economy $\{u, e, R\}$ in elementary securities is economy $\left\{u, e, \mathbb{I}_{S}\right\}$. For notational simplicity, we use lower-case characters for the corresponding (endogenous) variables in the representation in elementary securities:

DEFINITION 5. Given preferences and endowments $\{u, e\}$, a triple consisting of a vector of state prices, a profile of revenue transfers across states of the world and a profile of state-price impact matrices, $(\bar{p}, \bar{\theta}, \bar{m})$ is an equilibrium in elementary securities for $\{u, e\}$ if it is an equilibrium in thin financial markets for economy $\left\{u, e, \mathbb{I}_{S}\right\}$.

The following result says that, under our assumptions, equilibria in elementary securities exist and are (generically) locally unique. 
Proposition 3. Suppose there are at least three traders in the market. Then,

1. For any profile of preferences and endowments, $\{u, e\}$, there exists an equilibrium in elementary securities $(\bar{p}, \bar{\theta}, \bar{m})$ such that all price impact matrices $\bar{m}^{i}$ are diagonal and positive definite.

2. If, moreover, all preferences are three times continuously differentiable, then, generically in the space of preferences and endowments, all equilibria in elementary securities with diagonal, positive-definite price impact matrices are locally unique (i.e., isolated from one another). ${ }^{15}$

Proof: These results follow, respectively, from Theorems 1 and 2 in [12]. For the sake of simplicity and completeness, Appendix A2 specializes the proofs to the case considered here.

Q.E.D.

It is natural to ask whether in the equilibria resulting from Proposition 3 there are arbitrage opportunities and irrelevant price impacts. Observe that in the economy with elementary securities the only portfolio that gives zero return in all states is a zero portfolio, and obviously both the cost of such portfolio and its price impact are zero, regardless of the specific prices and price impact matrices. Therefore, equilibrium prices do not allow for weak arbitrage opportunities and there is no irrelevant price impact in the economy with elementary securities. The question of whether the no-arbitrage condition holds in the strong sense (so that a strong pricing kernel exists) is addressed in the next proposition.

Proposition 4. Let $(\bar{p}, \bar{\theta}, \bar{m})$ be an equilibrium in elementary securities for $\{u, e\}$, with diagonal, positive definite price impact matrices $\bar{m}^{i}$. Then, prices $\bar{p}$ allow no-arbitrage opportunities: $\bar{p} \gg 0$

Proof: For every trader $i$, it must be true that $\bar{p}=\partial u^{i}\left(e^{i}+\bar{\theta}^{i}\right)-\bar{m}^{i} \bar{\theta}^{i}$. For asset (state) $s$, since $\bar{m}^{i}$ is diagonal, the latter means that $\bar{p}_{s}=\partial u_{s}^{i}\left(e_{s}^{i}+\bar{\theta}_{s}^{i}\right)-\bar{m}_{s, s}^{i} \bar{\theta}_{s}^{i}$. By market clearing, there must exist $i$ for whom $\bar{\theta}_{s}^{i} \leq 0$, which means, by monotonicity of $u_{s}^{i}$ and positive definiteness of $\bar{m}^{i}$, that $\bar{p}_{s}>0$.

Q.E.D.

Critically, the implication of Proposition 4 does not follow simply from the existence of one rational trader with monotone preferences; rather, it follows from the facts that all traders are acting optimally according to their von Neumann-Morgenstern utility functions, which are separable, and that all markets clear. ${ }^{16}$

\footnotetext{
15 The sense in which this result holds for a "typical economy" is established in detail in the proof.

16 [14] provides examples of economies with traders characterized by non-separable utility functions where one might observe negative prices for some states of the world, even with preferences that are strictly monotone. In such case, negative state prices would result from cross-market effects associated with market power.
} 


\subsection{Equivalence of the Representations}

We say that a pair of portfolios $\left(\Theta^{i}, \theta^{i}\right) \in \mathbb{R}^{A} \times \mathbb{R}^{S}$ is $R$-associated if the two portfolios are payoff-equivalent, $R \Theta^{i}=\theta^{i}$. By market completeness, for every $\theta^{i}$ there is at least one $\Theta^{i}$ such that $\left(\Theta^{i}, \theta^{i}\right)$ is $R$-associated, for example

$$
\Theta^{i}=\left(\begin{array}{c}
R_{S}^{-1} \theta^{i} \\
0
\end{array}\right) .
$$

When there are no redundant assets, such $\Theta^{i}$ is unique.

Furthermore, we say that a triple of a vector of prices, a profile of portfolios and a profile of price impact matrices in market $R$, and a corresponding triple in elementary securities are $R$-associated if so are each of their components: $(P, \Theta, M)$ and $(p, \theta, m)$ are $R$-associated if:

1. the pair of prices $(P, p)$ is $R$-associated: $P=R^{\top} p$;

2. for each $i$, the pair of portfolios $\left(\Theta^{i}, \theta^{i}\right)$ is $R$-associated: $R \Theta^{i}=\theta^{i}$; and

3. for each $i$, the pair of price impact matrices $\left(M^{i}, m^{i}\right)$ is $R$-associated: $M^{i}=R^{\top} m^{i} R$.

The next lemma establishes the connection between the equilibria in economies with arbitrary assets and their counterparts with elementary securities.

Lemma 5. Suppose that $(\bar{P}, \bar{\Theta}, \bar{M})$ and $(\bar{p}, \bar{\theta}, \bar{m})$ are $R$-associated. Then,

1. if $(\bar{p}, \bar{\theta}, \bar{m})$ is an equilibrium in elementary securities for $\{u, e\}$ and $\sum_{i} \bar{\Theta}^{i}=0$, then $(\bar{P}, \bar{\Theta}, \bar{M})$ is an equilibrium in thin financial markets for $\{u, e, R\}$;

2. if $(\bar{P}, \bar{\Theta}, \bar{M})$ is an equilibrium in thin financial markets for $\{u, e, R\}$, then $(\bar{p}, \bar{\theta}, \bar{m})$ is an equilibrium in elementary securities for $\{u, e\}$.

Proof: See Appendix A1.

Q.E.D.

In the following sections we explore the theoretical and practical implications of this result.

\subsection{Equilibria With No-Arbitrage}

Suppose that trader $i$ has chosen a portfolio $\Theta^{i}$, even though an arbitrage opportunity $\Theta$ exists in the market. If $i$ were competitive, portfolio $\Theta^{i}+\lambda \Theta$, for any $\lambda>0$, would give her more utility. This need not be the case, though, when $i$ is noncompetitve, for her demand for portfolio $\Theta$, the arbitrage opportunity, may exert 'detrimental' effects of the price of $\Theta$ itself, and on the cost of portfolio $\Theta^{i}$; while the first effect is of second order, and hence vanishes for small $\lambda$, the second effect is of first order.

Proposition 4 has given a condition under which no-arbitrage remains a necessary condition of equilibria under noncompetitive behavior, for the case of a full set of elementary 
securities. The following result is the most important one in the paper: it shows that in economies with complete markets and separable preferences, noncompetitive behavior is not incompatible with the existence of a pricing kernel.

TheOREm 1. Suppose that there are at least three traders in the market. Then,

1. For any economy $\{u, e, R\}$, there exists an equilibrium $(\bar{P}, \bar{\Theta}, \bar{M})$ satisfying that

(a) all price impact matrices $\bar{M}^{i}$ are symmetric, positive definite and give no irrelevant price impact; and

(b) prices $\bar{P}$ allow no strong (and, hence, no weak) arbitrage opportunities, and a classical pricing kernel exists.

2. If, moreover, all individual preferences are three times continuously differentiable, then, for a generic profile of preferences and endowments $\{u, e\}$, and for any asset structure $R$, equilibria satisfying conditions (a) and (b) above are locally unique, at least up to the payoff equivalence of the portfolios: generically in $\{u, e\}$, every equilibrium $(\bar{P}, \bar{\Theta}, \bar{M})$ of $\{u, e, R\}$ has an open neighborhood such that if $(\tilde{P}, \tilde{\Theta}, \tilde{M})$ is an equilibrium for the same economy and lies in that open neighborhood, then $\tilde{P}=\bar{P}, \tilde{M}=\bar{M}$ and $R \tilde{\Theta}^{i}=R \bar{\Theta}^{i}$ for every trader $i$.

Proof: Part 1 follows from part 1 of Proposition 3: Given preferences and endowments $\{u, e\}$, it follows from the proposition that there exists an equilibrium in elementary securities $(\bar{p}, \bar{\theta}, \bar{m})$, and that in this equilibrium all price impact matrices $\bar{m}^{i}$ are diagonal. Define $\bar{P}=R^{\top} \bar{p}$ and, for all trader $i$, let

$$
\bar{\Theta}^{i}=\left(\begin{array}{c}
R_{S}^{-1} \bar{\theta}^{i} \\
0
\end{array}\right)
$$

and $\bar{M}^{i}=R^{\top} \bar{m}^{i} R$. By construction, $(\bar{P}, \bar{\Theta}, \bar{M})$ and $(\bar{p}, \bar{\theta}, \bar{m})$ are $R$-associated and $\sum_{i} \bar{\Theta}^{i}=0$. It follows from part 1 of Proposition 5 that $(\bar{P}, \bar{\Theta}, \bar{M})$ is an equilibrium in thin financial markets for $\{u, e, R\}$. By construction, every price impact matrix $\bar{M}^{i}$ gives no irrelevant price impact, and hence, it follows from Proposition 1 that $P$ allows no weak arbitrage opportunities. Moreover, since every matrix $\bar{m}^{i}$ is diagonal, it follows from part 1 of Proposition 4 that $\bar{p} \gg 0$, and, hence, that $\bar{P}=R^{\top} \bar{p}$ allows no strong arbitrage opportunities either.

For part 2, take any profile of preferences and endowments, $\{u, e\}$, in the generic set given by part 2 of Proposition 3, and fix any $R$ satisfying the assumptions imposed in the paper. Suppose, by way of contradiction, that there exist an equilibrium $(\bar{P}, \bar{\Theta}, \bar{M})$ and a sequence of equilibria $\left(P_{n}, \Theta_{n}, M_{n}\right)_{n=1}^{\infty}$, all of them satisfying conditions (a) and (b), such that

1. for every $n,\left(P_{n},\left(R \Theta_{n}^{i}\right)_{i=1}^{I}, M_{n}\right) \neq\left(\bar{P},\left(R \bar{\Theta}^{i}\right)_{i=1}^{I}, \bar{M}\right)$; and 
2. sequence $\left(P_{n}, \Theta_{n}, M_{n}\right)_{n=1}^{\infty}$ converges to $(\bar{P}, \bar{\Theta}, \bar{M})$.

Define $\bar{p}$ and $p_{n}$ to be the unique state-price vectors $R$-associated, respectively, to $\bar{P}$ and $P_{n}$. Similarly, let $\bar{m}$ and $m_{n}$ be the unique profiles of price impact matrices that are $R$-associated, respectively, to $\bar{M}$ and $M_{n}$. By part 2 of Lemma 5 , it follows that $\left(\bar{p},\left(R \bar{\Theta}^{i}\right)_{i=1}^{I}, \bar{m}\right)$ and all $\left(p_{n},\left(R \Theta_{n}^{i}\right)_{i=1}^{I}, m_{n}\right)$ are equilibria in elementary securities for $\{u, e\}$. Since $R$-association of prices and price impact matrices both define bijections, it follows from the first property above that for every $n,\left(p_{n},\left(R \Theta_{n}^{i}\right)_{i=1}^{I}, m_{n}\right) \neq\left(\bar{p},\left(R \bar{\Theta}^{i}\right)_{i=1}^{I}, \bar{m}\right)$. By the second property above, $\left(p_{n},\left(R \Theta_{n}^{i}\right)_{i=1}^{I}, m_{n}\right)$ converges to $\left(\bar{p},\left(R \bar{\Theta}^{i}\right)_{i=1}^{I}, \bar{m}\right)$, but this is impossible since all equilibria in elementary securities for $\{u, e\}$ are locally unique. Q.E.D.

\section{Thin Financial Markets and Asset Pricing}

\subsection{The Fundamental Theorem of Asset Pricing}

The following result is an extension of the Fundamental Theorem of Asset Pricing. It says the introduction of an asset whose payoff can be replicated by a combination of existing assets need not perturb non-competitive equilibria: the new asset is priced at the cost of the portfolio that replicates it, and it is not traded. What is new, though, is that the same principle by which the asset is priced applies to the price impacts it exerts and receives.

Theorem 2. Suppose that $(P, \Theta, M)$ is an equilibrium in thin financial markets for economy $\{u, e, R\}$, and that all price impact matrices $M^{i}$ give no irrelevant price impact. Let $\rho$ be a collection of new assets, taken as an $S \times \tilde{A}$ matrix, let $\tilde{R}=(R, \rho)$ be a new asset structure, and let

$$
\Theta_{\rho}=\left(\begin{array}{c}
R_{S}^{-1} \rho \\
0
\end{array}\right)
$$

be a collection of portfolios that replicate the assets in $\rho .{ }^{17}$ Then, an equilibrium for economy $\{u, e, \tilde{R}\}$ is given by $(\tilde{P}, \tilde{\Theta}, \tilde{M})$ with prices

$$
\tilde{P}=\left(\begin{array}{c}
P \\
\Theta_{\rho}^{\top} P^{\top}
\end{array}\right),
$$

individual portfolios $\tilde{\Theta}^{i}=\left(\left(\Theta^{i}\right)^{\top}, 0\right)^{\top}$, and individual price impact matrices

$$
\tilde{M}^{i}=\left(\begin{array}{cc}
M^{i} & M^{i} \Theta_{\rho} \\
\Theta_{\rho}^{\top} M^{i} & \Theta_{\rho}^{\top} M^{i} \Theta_{\rho}
\end{array}\right) .
$$

Proof: By Corollary 1, there exists $p \in \mathbb{R}^{S}$ such that $R^{\top} p=P$. By construction, then, $p=\left(R_{S}^{\top}\right)^{-1}\left(P_{1}, \ldots, P_{S}\right)^{\top}$. Define also, for each $i, \theta^{i}=R \Theta^{i}$ and $m^{i}=\left(R_{S}^{\top}\right)^{-1} M_{S}^{i} R_{S}^{-1}$. By

\footnotetext{
17 Notice that $\Theta_{\rho}$ is an $A \times \tilde{A}$ matrix, and not just a column vector. The $a$-th column in this matrix replicates the $a$-th payoff vector in $\rho$.
} 
Lemma $3,(P, \Theta, M)$ and $(p, \theta, m)$ are $R$-associated, and, in particular,

$$
M^{i}=R^{\top} m^{i} R=\left(\begin{array}{cc}
M_{S}^{i} & M_{S}^{i} R_{S}^{-1} R_{A-S} \\
R_{A-S}^{\top}\left(R_{S}^{-1}\right)^{\top} M_{S}^{i} & R_{A-S}^{\top}\left(R_{S}^{-1}\right)^{\top} M_{S}^{i} R_{S}^{-1} R_{A-S}
\end{array}\right) .
$$

It follows from part 2 of Proposition 5 that $(p, \theta, m)$ is an equilibrium in elementary securities for $\{u, e\}$. Also by construction, prices $(\tilde{P}, p)$ and portfolios $(\tilde{\Theta}, \theta)$ are $\tilde{R}$ associated. Moreover, for each trader $i$,

$$
\tilde{R}^{\top} m^{i} \tilde{R}=\left(\begin{array}{cc}
M^{i} & R^{\top} m^{i} \rho \\
\rho^{\top} m^{i} R & \rho^{\top} m^{i} \rho
\end{array}\right)
$$

By direct computation,

$$
R^{\top} m^{i} \rho=\left(\begin{array}{c}
M_{S}^{i} R_{S}^{-1} \rho \\
R_{A-S}^{\top}\left(R_{S}^{\top}\right)^{-1} M_{S}^{i} R_{S}^{-1} \rho
\end{array}\right)=M^{i} \Theta_{\rho},
$$

and $\rho^{\top} m^{i} \rho=\Theta_{\rho}^{\top} M^{i} \Theta_{\rho}$, so $\tilde{R}^{\top} m^{i} \tilde{R}=\tilde{M}^{i}$. It follows that $(\tilde{M}, m)$ is $\tilde{R}$-associated, and the result then follows from part 1 of Proposition 5.

Corollary 1 and Proposition 2 can be used to write the equilibrium prices for the economy endowed with asset structure $\tilde{R}$ as

$$
\tilde{P}=\tilde{R}^{\top} p\left(\begin{array}{c}
P \\
\rho^{\top} p
\end{array}\right) .
$$

for some $p \in \mathbb{R}^{S}$. It is in this sense that vector $p$ obtained from corollary is a pricing kernel: the prices of all assets, including derivatives that can be replicated from existing assets, can be expressed as the value of their returns at state prices $p$. Crucially, Proposition 1 established the existence of equilibria in which $p$ is a pricing kernel in the classical sense $(p \gg 0)$ and can be used to define a probability measure over states of the world.

Note also that the computation of price impact matrices can be obtained via their analogous in elementary securities as well: this is the intuition of equation (19). In this sense, one can view the price impact matrices in elementary securities as "price impact kernels."

\subsection{The Interest Rate Puzzle}

It follows from the Fundamental Theorem of Asset Pricing that the return to a riskless asset can be computed as the inverse of the sum of all state prices. The next proposition shows that if one assumes that all individuals have identical CRRA Bernoulli utility functions, ${ }^{18}$ then trade in financial markets results in an risk-free interest rate that is below the one from a competitive model.

\footnotetext{
18 Namely, if for all $i, u_{s}^{i}\left(c_{s}^{i}\right)=\left(c_{s}^{i}\right)^{1-\phi_{s}} /\left(1-\phi_{s}\right)$, where $\phi_{s}>0$ is the coefficient of risk aversion. For the result that follows, the coefficient of risk aversion can change across states, but is the same for all individuals, given a state.
} 
Proposition 5. Consider an economy $\{u, e, R\}$, where all individuals have identical CRRA Bernoulli utility functions. In any equilibrium satisfying the two properties stated in part 1 on Theorem 1, the risk-free interest rate is always below the rate that would arise in a competitive economy.

Proof: The third derivative of a CRRA utility function is positive, and, hence, the assumptions of Proposition 4 in [12] hold. Then, state-by-state, the equilibrium price of an elementary security is above its competitive counterpart and therefore the risk-free interest rate is lower than in the corresponding competitive market.

Q.E.D.

It follows that when financial markets are thin, using the competitive model to explain the empirical returns will lead to a risk-free interest rate puzzle. ${ }^{19}$ Intuitively, the reason is that, while in a competitive model with identical quasilinear preferences the consumption of all agents is the same, in thin markets traders do not perfectly hedge their risks, and hence equilibrium consumption is not degenerate; with convex marginal utility, ${ }^{20}$ in each state the same per capita consumption is associated with a higher average marginal utility across all traders, which enhances the price of an elementary security lowers the risk-free interest rate. Our finding, however, should be interpreted with care, as we did not assess the quantitative relevance of the mechanism, which requires a fully-fledged dynamic model of thin markets. ${ }^{21}$

Finally, notice that this model of thin financial markets is consistent with the ubiquitous observation that that a dollar invested in a small-cap stock gives a significantly higher expected return than the same dollar invested in a large-cap company, ceteris paribus: since large-cap stocks are traded in deeper markets, the difference in returns is a premium for market depth (or liquidity). Such premium cannot be reconciled with the competitive theory, while it naturally arises in the model of thin trading.

\section{A (Very) Simple Example}

We now use a "coconut" example to illustrate numerical differences between equilibria in competitive economies and equilibria in thin financial markets. We use this computations to illustrate that noncompetitiveness may help in explaining well-known asset pricing puzzles, such as the risk-free interest rate puzzle. Finally we show how Theorem 2 can be applied to price derivatives in thin markets.

\footnotetext{
19 This is true even if the specified CRRA preferences have a "reasonable" risk-aversion parameter.

20 The third derivative of a CRRA utility function is positive, which implies that in each state a marginal utility is convex.

21 Until now the only dynamic model of thin trading, proposed in [16], assumes quadratic utility functions, whereas the considered return biases require convex marginal utility.
} 


\subsection{The ECONOMY}

Consider an island where the only production technology available is coconut palms. There are three equally likely states of the world, $s=1,2,3$ : in state 1 there is a drought in the north of the island and the palms there are not productive; in state 2 , the drought is in the south of the island; in state 3, there is no drought anywhere in the island, and in this case each palm produces two coconuts.

There are two types of individual, $t=1,2$, and there are two (identical) individuals of each type. Type-1 individuals live in the north, so they have wealth only when that region of the island is productive: they are endowed with $e^{1}=(0,2,2)$. Type-2 individuals live in the south, and, analogously, they have $e^{2}=(2,0,2)$. All four individuals have identical preferences,

$$
u(c)=c_{0}+\frac{1}{3} \sum_{s} \ln \left(c_{s}\right) .
$$

There are three securities that can be traded in this economy: a riskless bond, $R^{1}=$ $(1,1,1)^{\top}$, a share in a coconut palm from the south, $R^{2}=(0,2,2)^{\top}$, and a share in a coconut palm from the north, $R^{3}=(2,0,2)^{\top}$.

\subsection{Equilibrium in Thin Financial Markets}

If all traders ignore their price effects and behave competitively, the equilibrium in these financial markets is at prices $\tilde{P}=(5 / 6,1,1)^{\top}$, with both type- 1 individuals buying a portfolio $\tilde{\Theta}^{1}=(0,-1 / 2,1 / 2)^{\top}$ and both type- 2 individuals selling that portfolio.

\subsubsection{Elementary SECURIties}

For simplicity of computation, we can solve first for an equilibrium in elementary securities, and then use Lemma 5 to obtain one in the original thin financial markets. By numerical computation, an equilibrium in elementary securities is as follows: ${ }^{22}$

1. prices are $\bar{p}=(0.3920,0.3920,0.1667)^{\top}$;

2 . both type- 1 individuals buy a portfolio $\bar{\theta}^{1}=(0.6647,-0.6647,0)$ and estimate a price impact matrix

$$
\bar{m}^{1}=\left(\begin{array}{ccc}
0.1646 & 0 & 0 \\
0 & 0.2142 & 0 \\
0 & 0 & 0.0417
\end{array}\right)
$$

22 This equilibrium can be compared with the competitive equilibrium of this economy in the case of a full set or elementary securities: prices would be $\tilde{p}=(1 / 3,1 / 3,1 / 6)^{\top}$, type-1 individuals would buy $\tilde{\theta}^{1}=(1,-1,0)^{\top}$, which would be sold by type- 2 individuals. 
3. each type-2 individual sells the portfolio purchased by a type- 1 individual, $\bar{\theta}^{2}=-\bar{\theta}^{1}$, and they both estimate a price impact matrix

$$
\bar{m}^{2}=\left(\begin{array}{ccc}
0.2142 & 0 & 0 \\
0 & 0.1646 & 0 \\
0 & 0 & 0.0417
\end{array}\right) .
$$

\subsubsection{EqUiLIBRIUM}

We can now use Lemma 5 to compute the equilibrium with asset structure $R=\left(R^{1}, R^{2}, R^{3}\right)$ :

1. prices are given by

$$
\bar{P}=R^{\top} \bar{p}=(0.9507,1.1173,1.1173)^{\top}
$$

2. each individual of type 1 buys a portfolio

$$
\bar{\Theta}^{1}=R^{-1} \bar{\theta}^{1}=\left(\begin{array}{c}
0.0000 \\
-0.3324 \\
0.3324
\end{array}\right)
$$

which is sold by a type-2 individual: $\bar{\Theta}^{2}=-\bar{\Theta}^{1}$; and

3. each individual of type 1 estimates that her price impacts are

$$
\bar{M}^{1}=R^{\top} \bar{m}^{1} R=\left(\begin{array}{lll}
0.4205 & 0.5117 & 0.4126 \\
0.5117 & 1.0233 & 0.1667 \\
0.4126 & 0.1667 & 0.8252
\end{array}\right)
$$

while each individual of type 2 estimates

$$
\bar{M}^{2}=R^{\top} \bar{m}^{2} R=\left(\begin{array}{lll}
0.4205 & 0.4126 & 0.5117 \\
0.4126 & 0.8252 & 0.1667 \\
0.5117 & 0.1667 & 1.0233
\end{array}\right)
$$

One can readily verify that these values satisfy all the conditions of Lemma 1, and, hence, constitute an equilibrium for $\{u, e, R\}$.

\subsection{Price Biases}

Notice that in this economy the competitive risk-free interest rate is $\tilde{r}=1 / \tilde{P}_{1}-1=0.2$. Importantly, notice that when these agents recognize their price impacts the risk-free interest rate is lower: it is $\bar{r}=1 / \bar{P}_{1}-1=0.0519$.

Another anomaly robustly documented in the empirical literature is the equity premium puzzle. In our example, if trade were competitive the equity premium would be, for asset 2 ,

$$
\frac{E\left(R^{2}\right)}{\tilde{P}_{2}}-\tilde{r}=0.1333
$$


In the equilibrium in thin markets, the same return is traded at a higher premium

$$
\frac{E\left(R^{2}\right)}{\bar{P}_{2}}-\bar{r}=0.1414
$$

Therefore in thin markets the equity premium is significantly above the one obtained in the competitive setting. Intuitively, price impacts of the traders are the highest for elementary securities corresponding to "bad" states of the economy, and for such states the price biases are strongest.

\subsection{Arbitrage Pricing in Practice}

To the existing assets, a bond and two stocks, we add call options shares of the palms, with strike prices equal to 1 in both cases. These assets can be written as

$$
R^{4}=\left(\begin{array}{c}
\max \left\{R_{1}^{2}-1,0\right\} \\
\max \left\{R_{2}^{2}-1,0\right\} \\
\max \left\{R_{3}^{2}-1,0\right\}
\end{array}\right)=\left(\begin{array}{l}
0 \\
1 \\
1
\end{array}\right),
$$

and

$$
R^{5}=\left(\begin{array}{c}
\max \left\{R_{1}^{3}-1,0\right\} \\
\max \left\{R_{2}^{3}-1,0\right\} \\
\max \left\{R_{3}^{3}-1,0\right\}
\end{array}\right)=\left(\begin{array}{l}
1 \\
0 \\
1
\end{array}\right) .
$$

Let $\rho=\left(R^{4}, R^{5}\right)$, and notice that these claims are reproduced by

$$
\Theta_{\rho}=\left(\begin{array}{cc}
0 & 0 \\
1 / 2 & 0 \\
0 & 1 / 2
\end{array}\right) \text {. }
$$

By Theorem 2, we can obtain an equilibrium where prices of the existing (primitive) assets remain the same, all traders constitute the same portfolios as before (that is, they do not trade the options), the options are priced at $\bar{P} \Theta_{\rho}=(0.5587,0.5587)$, and the price impact matrices are as follows: for each type-1 individual the matrix is

$$
\left(\begin{array}{cc}
\bar{M}^{1} & \bar{M}^{1} \Theta_{\rho} \\
\Theta_{\rho}^{\top} \bar{M}^{1} & \Theta_{\rho}^{\top} \bar{M}^{1} \Theta_{\rho}
\end{array}\right)=\left(\begin{array}{ccccc}
0.4205 & 0.5117 & 0.4126 & 0.2558 & 0.2063 \\
0.5117 & 1.0233 & 0.1667 & 0.5117 & 0.0833 \\
0.4126 & 0.1667 & 0.8252 & 0.0833 & 0.4126 \\
0.2558 & 0.5117 & 0.0833 & 0.2558 & 0.0417 \\
0.2063 & 0.0833 & 0.4126 & 0.0417 & 0.2063
\end{array}\right),
$$

while for each type-2 individual it is

$$
\left(\begin{array}{cc}
\bar{M}^{2} & \bar{M}^{2} \Theta_{\rho} \\
\Theta_{\rho}^{\top} \bar{M}^{2} & \Theta_{\rho}^{\top} \bar{M}^{2} \Theta_{\rho}
\end{array}\right)=\left(\begin{array}{ccccc}
0.4205 & 0.4126 & 0.5117 & 0.2063 & 0.2558 \\
0.4126 & 0.8252 & 0.1667 & 0.4126 & 0.0833 \\
0.5117 & 0.1667 & 1.0233 & 0.0833 & 0.5117 \\
0.2063 & 0.4126 & 0.0833 & 0.2063 & 0.0417 \\
0.2558 & 0.0833 & 0.5117 & 0.0417 & 0.2558
\end{array}\right)
$$




\section{Concluding Remarks}

We have studied a model of trade in financial markets where individual investors recognize the fact that prices do depend on their trades. In this setting, the argument why asset prices do not allow for arbitrage opportunities at equilibrium fails. In the customary argument, arbitrage opportunities cannot exist because if they do, the portfolios that individuals are demanding cannot be optimal: whatever her preferences and beliefs ${ }^{23}$ adding one unit of the arbitrage opportunity to the portfolio of one trader would make her strictly better-off. Thus, according to this argument, equilibrium asset prices eliminate arbitrage opportunities and embed, in consequence, an objective probability distribution that allows for the pricing of any state-contingent claim in the economy to be computed as the discounted expected return it entails. But, indeed, this argument is untenable if individual investors do anticipate that their trades will affect asset prices: even if an arbitrage opportunity exists, it may be that an investor's attempt to exploit it affects the prices in a way such that the cost of her existing portfolio increases to the point that she is no longer better-off in ex-ante terms. If a theory of asset pricing based on an objective probability embedded in prices is going to be consistent with noncompetitive behavior of investors, a new argument has to be provided.

In this paper, we have tackled that question in the context of a two-period, financial economy with a finite number of states of the world. We have considered a situation in which all traders know that, given a status quo of prices and trades in the market, if they were to attempt a different trade, they would (have to) affect prices in order to guarantee that the rest of the market is willing to accommodate their increased demands or sales. In such a case, the predictions of models where all traders follow price-taking behavior are inapplicable. For instance, each individual's willingness to trade is lower than in the competitive case, and, consequently, not all gains to trade are exhausted and trade leads to an equilibrium in which the asset allocation is Pareto-inefficient. ${ }^{24}$ Here, we have shown that, even in noncompetitive financial markets, financial equilibria that preclude arbitrage opportunities do exist.

Some assumptions were made. Firstly, we only consider agents with preferences that have von Neumann-Morgenstern representation with respect to future consumption, and that are quasilinear with respect to date-zero consumption, a variable in which we impose no non-negativity constraints; effectively, these assumptions leave all future consumption free of income effects, and simplifies substitution effects across consumption in different states of the world, which makes our mathematical problem more tractable. Secondly,

\footnotetext{
${ }^{23}$ Assuming, of course, monotonicity of ex-ante preferences on consumption in all future states of the world.

24 As long as a priori there are some gains to trade, i.e. the initial endowments are Pareto inefficient. The deadweight loss due to non-competitive trading depends negatively on the depth of the market, and it vanishes completely as a number of traders approaches infinity (see [12]).
} 
we consider only the case in which the existing financial markets allow for complete insurance opportunities against risk; this allows us to obtain an auxiliary representation of the economy by replacing the financial markets with a complete set of elementary securities, in which, thanks to the separability of preferences, we can restrict attention to the case when price cross-effects are null: each agent beliefs, a posteriori correctly, that if she expands her order for some security, only the price of that security will be affected.

We then invoke the argument of [12] to prove the existence of equilibria in the auxiliary economy in which no arbitrage opportunities can exist. This conclusion, though, follows as a consequence of the facts that all traders are individually rational and all markets are clearing, which contrasts with the competitive case, in which the existence of just one individually rational trader suffices, regardless of market clearing. We then associate the equilibria of the auxiliary economy with equilibria on the original financial structure, and obtain that at these equilibria no arbitrage opportunities can exist either. Furthermore, we provide an extension of the fundamental theorem of asset pricing to (two-period, finite) noncompetitive economies, which allow not only for the computation of the prices of redundant securities, but also for the direct determination of the price impacts exerted by these securities (and also of those exerted by other securities on these). The effect of market thinness on asset prices is not clear without further assumptions. If one assumes a symmetric economy with CRRA preferences, state prices are above the ones that would prevail were the markets competitive. ${ }^{25}$

Critically, in our analysis market power is determined endogenously, as part of the definition of equilibrium. Here, we have considered the case in which traders correctly estimate their price impacts to a first-order level of accuracy: implicit in their individual portfolio problems, they impute a correct linear approximation to the real inverse demand they face from the rest of the market (which, in itself, depends on the estimations other traders are making of the inverse demand they face, estimations that depend themselves on the one made by the trader in question). This assumption simplifies the definition and treatment of equilibrium, in that it guarantees that each trader's solution to her portfolio problem is characterized by its first-order conditions, but is of no further importance. Moreover, [13] provides strategic foundations for the equilibrium used here.

The extension of these results, and the relaxation of the assumptions made here remain topics for further research. If one guarantees that the second-order conditions of the portfolio problem are satisfied for each trader, then the assumption that they only estimate their inverse demands to a first-order level of accuracy can be removed. The assumptions of separability and quasilinearity are useful, but ought to be relaxed. Most critically, however, the question of whether our results hold true for economies with incomplete financial markets remains open.

\footnotetext{
${ }^{25}$ For the detailed study of model predictions for different market structures and utility functions, see [12].
} 


\section{Appendix A1: Lemmata}

Proof of Lemma 1: For necessity, the first two conditions are immediate, and we only need to concentrate on the third condition.

For any investor $j$, since $\Theta^{j}$ is stable given $\left(\bar{P}, \bar{M}^{j}\right)$ it must be that it solves the equation $\bar{P}=R^{\top} \partial u^{j}\left(e^{j}+R \bar{\Theta}^{j}\right)-\bar{M}^{j} \bar{\Theta}^{j}$. By strong concavity of preferences, nonredundancy of assets and positive definiteness of the price impact matrix, this defines, locally around $\bar{P}$, a differentiable individual (stable) demand function $\Theta^{j}\left(P ; \bar{M}^{j}\right)$, with derivative

$$
\partial_{P} \Theta^{j}\left(\bar{P} ; \bar{M}^{j}\right)=\left(R^{\top} \partial^{2} u^{j}\left(e^{j}+R \bar{\Theta}^{j}\right) R-\bar{M}^{j}\right)^{-1},
$$

a negative definite matrix.

Thus, the demand trader $i$ faces from the rest of the market is, locally around $\bar{P}, \varphi^{i}(P)=$ $\sum_{j \neq i} \Theta^{j}\left(P ; \bar{M}^{j}\right)$. By construction,

$$
\partial \varphi^{i}(\bar{P})=\sum_{j \neq i}\left(R^{\top} \partial^{2} u^{j}\left(e^{j}+R \bar{\Theta}^{j}\right) R-\bar{M}^{j}\right)^{-1}
$$

again a negative definite matrix. A subequilibrium triggered by a local deviation $\tilde{\Theta}^{i}$ (close enough to $\left.\bar{\Theta}^{i}\right)$ requires prices $\tilde{P}$ such that $\varphi^{i}(\tilde{P})=-\tilde{\Theta}^{i}$. This defines, locally, a differentiable subequilibrium price function $\phi^{i}\left(\tilde{\Theta}^{i}\right)$, with the property that

$$
\partial \phi^{i}\left(\bar{\Theta}^{i}\right)=-\left(\partial \varphi^{i}(\bar{P})\right)^{-1}=\mathcal{H}\left(\left(\bar{M}^{j}-R^{\top} \partial^{2} u^{j}\left(e^{j}+R \bar{\Theta}^{j}\right) R\right)_{j \neq i}\right) .
$$

Now, since $\bar{M}$ is mutually consistent given $(\bar{P}, \bar{\Theta})$, there exists a local inverse demand function $P^{i}$. Since subequilibria are locally unique, it must be that, in a neighborhood of $\bar{\Theta}^{i}$, $\phi^{i}\left(\tilde{\Theta}^{i}\right)=P^{i}\left(\tilde{\Theta}^{i}\right)$, which immediately implies, again by mutual consistency of $M$, that

$$
M^{i}=\partial P^{i}\left(\bar{\Theta}^{i}\right)=\partial \phi^{i}\left(\bar{\Theta}^{i}\right)=\mathcal{H}\left(\left(M^{j}-R^{\top} \partial^{2} u^{j}\left(e^{j}+R \bar{\Theta}^{j}\right) R\right)_{j \neq i}\right) .
$$

For sufficiency, market clearing and stability of all trades are immediate, and we only need to prove mutual consistency of profile $\bar{M}$.

As in the proof of necessity, by the second condition of the lemma, for each trader $i$ we again have, locally around $\bar{P}$, an inverse demand function $\varphi^{i}(P)=\sum_{j \neq i} \Theta^{j}\left(P ; \bar{M}^{j}\right)$, with

$$
\partial \varphi^{i}(\bar{P})=\sum_{j \neq i}\left(R^{\top} \partial^{2} u^{j}\left(e^{j}+R \bar{\Theta}^{j}\right) R-\bar{M}^{j}\right)^{-1}
$$

a negative definite matrix. By the implicit function theorem we can find neighborhoods $N^{i}\left(\bar{\Theta}^{i}\right)$ and $N^{i}(\bar{P})$, and a diffeomorphism $\rho^{i}: N^{i}\left(\bar{\Theta}^{i}\right) \rightarrow N^{i}(\bar{P})$, such that

(a) $P^{i}\left(\tilde{\Theta}^{i}\right)$ is the only $P$ in $N^{i}(\bar{P})$ satisfying that $\varphi^{i}(P)+\tilde{\Theta}^{i}=0$; and

(b) $\partial P^{i}\left(\bar{\Theta}^{i}\right)=-\left(\sum_{j \neq i}\left(R^{\top} \partial^{2} u^{j}\left(e^{j}+R \bar{\Theta}^{j}\right) R-\bar{M}^{j}\right)^{-1}\right)^{-1}$.

By property (a), for every $\tilde{\Theta}^{i} \in N\left(\bar{\Theta}^{i}\right), \mathcal{P}^{i}\left(\tilde{\Theta}^{i} ; M^{-i}\right) \cap N(\bar{P})=\left\{P^{i}\left(\tilde{\theta}^{i}\right)\right\}$, while, by property (b) and the third condition of the lemma, $\partial P^{i}\left(\bar{\Theta}^{i}\right)=M^{i}$.

Q.E.D.

Proof of Lemma 2: That prices in the set allow no weak arbitrage opportunities is straightforward. Now, vector $P$ allows no weak arbitrage opportunities if, and only if, there exists no $\Theta$ for which $P \cdot \Theta<0$ and $R \Theta=0$. It follows from Farkas's Lemma that if $P$ allows no weak arbitrage opportunities, then for some $\left(p_{0}, p_{1}\right) \in \mathbb{R}_{++} \times \mathbb{R}^{S}$, it is true that $p_{0} P=R^{\top} p_{1}$. Letting $p=\frac{1}{p_{0}} p_{1}$ completes the proof.

Q.E.D. 
Proof of Lemma 3: Write

$$
M=\left(\begin{array}{cc}
M_{S} & \Delta \\
\Delta^{\top} & \Gamma
\end{array}\right)
$$

Define

$$
T=\left(\begin{array}{c}
-R_{S}^{-1} R_{A-S} \\
\mathbb{I}_{A-S}
\end{array}\right)
$$

and notice that $R T=0$. Since $M$ gives no irrelevant impact, it follows that $M T=0$, which implies that $M_{S} R_{S}^{-1} R_{A-S}=\Delta$ and $\Delta^{\top} R_{S}^{-1} R_{A-S}=\Gamma$.

Now, by direct computation,

$$
R^{\top} m R=\left(\begin{array}{cc}
M_{S} & R_{S}^{\top} m R_{A-S} \\
R_{A-S}^{\top} m R_{S} & R_{A-S}^{\top} m R_{A-S}
\end{array}\right),
$$

whereas $R^{\top} m R T=0$, so $M_{S} R_{S}^{-1} R_{A-S}=R_{S}^{\top} m R_{A-S}$. Immediately, it follows that $R_{S}^{\top} m R_{A-S}=$ $\Delta$, which implies that $R_{A-S}^{\top} m R_{A-S}=\Delta^{\top} R_{S}^{-1} R_{A-S}=\Gamma$, and hence that $R^{\top} m R=M$.

For uniqueness, suppose that $R^{\top} \tilde{m} R=M$. Then,

$$
\tilde{m}=\left(R_{S}^{\top}\right)^{-1} R_{S}^{\top} \tilde{m} R_{S}\left(R_{S}\right)^{-1}=\left(R_{S}^{\top}\right)^{-1} M_{S}\left(R_{S}\right)^{-1}=m .
$$

Q.E.D.

Proof of Lemma 4: By construction,

$$
\sum_{j \neq i}\left(\tilde{\Theta}^{j}+(I-1)^{-1}\left(\tilde{\Theta}^{i}-\hat{\Theta}^{i}\right)\right)+\hat{\Theta}^{i}=\sum_{j \neq i} \tilde{\Theta}^{j}+\tilde{\Theta}^{i}-\hat{\Theta}^{i}+\hat{\Theta}^{i}=0
$$

and hence all markets clear. It only remains to show that for each $j \neq i$, trade $\tilde{\Theta}^{j}+(I-$ $1)^{-1}\left(\tilde{\Theta}^{i}-\hat{\Theta}^{i}\right)$ is stable for $j$ given $\left(\tilde{P}, \bar{M}^{j}\right)$. Suppose not: for some $j$ and some $\Theta^{j}$,

$$
\begin{aligned}
& -\left(\tilde{P}+\bar{M}^{j}\left(\Theta^{j}-\left(\tilde{\Theta}^{j}+(I-1)^{-1}\left(\tilde{\Theta}^{i}-\hat{\Theta}^{i}\right)\right)\right)\right) \cdot \Theta^{j}+u^{j}\left(e^{j}+R \Theta^{j}\right) \\
& >-\tilde{P} \cdot\left(\tilde{\Theta}^{j}+(I-1)^{-1}\left(\tilde{\Theta}^{i}-\hat{\Theta}^{i}\right)\right)+u^{j}\left(e^{j}+R\left(\tilde{\Theta}^{j}+(I-1)^{-1}\left(\tilde{\Theta}^{i}-\hat{\Theta}^{i}\right)\right)\right) .
\end{aligned}
$$

Since $R\left(\tilde{\Theta}^{i}-\hat{\Theta}^{i}\right)=0$ and $\bar{M}^{j}$ gives no irrelevant price impact, it follows that $\bar{M}^{j}\left(\tilde{\Theta}^{i}-\hat{\Theta}^{i}\right)=0$ and, by Proposition 1, $\tilde{P} \cdot\left(\tilde{\Theta}^{i}-\hat{\Theta}^{i}\right)=0$. Then, the previous equation is equivalent to

$$
-\left(\tilde{P}+\bar{M}^{j}\left(\Theta^{j}-\tilde{\Theta}^{j}\right)\right) \cdot \Theta^{j}+u^{j}\left(e^{j}+R \Theta^{j}\right)>-\tilde{P} \cdot \tilde{\Theta}^{j}+u^{j}\left(e^{j}+R \tilde{\Theta}^{j}\right),
$$

which means that $\tilde{\Theta}^{j}$ is not stable for $j$ given $\left(\tilde{P}, \bar{M}^{j}\right)$, contradicting the fact that $\left(\tilde{P}, \tilde{\Theta}^{-i}\right)$ is a subequilibrium triggered by trade $\tilde{\Theta}^{i}$.

Q.E.D.

Proof of Lemma 5: For notational definiteness, we denote by $\mathcal{S}^{i}\left(\Theta^{i} ; M^{-i}, R\right)$ and $\mathcal{P}^{i}\left(\Theta^{i} ; M^{-i}, R\right)$ the sets of subequilibria triggered by $\Theta^{i}$, given $M^{-i}$, and its projection into the space of prices, when the economy is endowed with asset structure $R$. We distinguish these sets for the case of elementary securities, by denoting them as $\mathcal{S}^{i}\left(\Theta^{i} ; M^{-i}, \mathbb{I}_{S}\right)$ and $\mathcal{P}^{i}\left(\Theta^{i} ; M^{-i}, \mathbb{I}_{S}\right)$.

Notice first that, since each $\left(\bar{M}^{i}, \bar{m}^{i}\right)$ is $R$-associated, it is immediate that all $\bar{M}^{i}$ give no irrelevant price impacts. Also, notice that, by Lemma $3, \bar{m}^{i}=\left(R_{S}^{-1}\right)^{\top} \bar{M}_{S}^{i} R_{S}^{-1}$ for all $i$.

For the first claim, suppose that $(\bar{p}, \bar{\theta}, \bar{m})$ is an equilibrium for $\left\{u, e, \mathbb{I}_{S}\right\}$ and $\sum_{i} \bar{\Theta}^{i}=0$. Market clearing is assumed, so we only need to show that each $\bar{\Theta}^{i}$ is stable for $i$ given $\left(\bar{P}, \bar{M}^{i}\right)$, and that the profile $\bar{M}$ is mutually consistent.

Suppose that $\bar{\Theta}^{i}$ is not stable for $i$ given $\left(\bar{P}, \bar{M}^{i}\right)$. Then, we can fix $\Theta$ such that

$$
-\left(\bar{P}+\bar{M}^{i}\left(\Theta-\bar{\Theta}^{i}\right)\right) \cdot \Theta+u^{i}\left(e^{i}+R \Theta\right)>-\bar{P} \cdot \bar{\Theta}^{i}+u^{i}\left(e^{i}+R \bar{\Theta}^{i}\right) .
$$


Given that $(\bar{P}, \bar{\Theta}, \bar{M})$ and $(\bar{p}, \bar{\theta}, \bar{m})$ are $R$-associated, it follows that

$$
-\left(R^{\top} \bar{p}+R^{\top} \bar{m}^{i} R\left(\Theta-\bar{\Theta}^{i}\right)\right) \cdot \Theta+u^{i}\left(e^{i}+R \Theta\right)>-\left(R^{\top} \bar{p}\right) \cdot \bar{\Theta}^{i}+u^{i}\left(e^{i}+R \bar{\Theta}^{i}\right),
$$

so, letting $\theta=R \Theta$, by direct computation,

$$
-\left(\bar{p}+\bar{m}^{i}\left(\theta-\bar{\theta}^{i}\right)\right) \cdot \theta+u^{i}\left(e^{i}+\theta\right)>-\bar{p} \cdot \bar{\theta}^{i}+u^{i}\left(e^{i}+\bar{\theta}^{i}\right),
$$

contradicting the fact that, in the economy with elementary securities, $\bar{\theta}^{i}$ is stable for $i$ given $\left(\bar{p}, \bar{m}^{i}\right)$.

Now, for each $i$, by mutual consistency of profile $\bar{m}$, there exist neighborhoods $N^{i}\left(\overline{\theta^{i}}\right)$ and $N^{i}(\bar{p})$ and a differentiable, local inverse demand function $p^{i}: N^{i}\left(\bar{\theta}^{i}\right) \rightarrow N^{i}(\bar{p})$ such that $p^{i}\left(\bar{\theta}^{i}\right)=\bar{p}$ and $\partial p^{i}\left(\bar{\theta}^{i}\right)=\bar{m}^{i}$. Then, we can define open neighborhoods

$$
N^{i}\left(\bar{\Theta}^{i}\right)=\left\{\Theta \mid R \Theta \in N^{i}\left(\bar{\theta}^{i}\right)\right\}
$$

and

$$
N^{i}(\bar{P})=\left\{P \mid\left(R_{S}^{\boldsymbol{\top}}\right)^{-1}\left(P_{1}, \ldots, P_{S}\right)^{\top} \in N^{i}(\bar{p})\right\} .
$$

By construction, $f(\Theta)=R \Theta$ maps $N^{i}\left(\bar{\Theta}^{i}\right)$ into $N^{i}\left(\bar{\theta}^{i}\right)$, and $g(p)=R^{\top} p$ maps $N^{i}(\bar{p})$ into $N^{i}(\bar{P})$, so we can define $P^{i}=g \circ p^{i} \circ f: N^{i}\left(\bar{\Theta}^{i}\right) \rightarrow N^{i}(\bar{P})$. First, notice that $P^{i}\left(\bar{\Theta}^{i}\right)=R^{\top} p^{i}\left(R \bar{\Theta}^{i}\right)=$ $R^{\top} \bar{p}=\bar{P}$, while

$$
\partial P^{i}\left(\bar{\Theta}^{i}\right)=R^{\top} \partial p^{i}\left(\bar{\theta}^{i}\right) R=R^{\top} \bar{m}^{i} R=\bar{M}^{i} .
$$

We now want to show that for any $\Theta \in N^{i}\left(\bar{\Theta}^{i}\right), P^{i}(\Theta)$ is the unique (locally) subequilibrium price triggered by $\Theta^{i}=\Theta$, namely that $\mathcal{P}^{i}\left(\Theta ; \bar{M}^{i}, R\right) \cap N^{i}(\bar{P})=\left\{P^{i}(\Theta)\right\}$. First, notice that in the economy with elementary securities, for some $\theta^{-i},\left(p^{i}(R \Theta), \theta^{-i}\right) \in \mathcal{S}^{i}\left(R \Theta ; \bar{m}^{-i}, \mathbb{I}_{S}\right)$. Defining

$$
\Theta^{j}=\left(\begin{array}{c}
R_{S}^{-1}\left(\theta^{j}+\frac{1}{I-1} R \Theta\right) \\
0
\end{array}\right)-\frac{1}{I-1} \Theta,
$$

for each $j \neq i$, it follows that $\left(P^{i}(\Theta),\left(\Theta^{j}\right)_{j \neq i}\right) \in \mathcal{S}^{i}\left(\Theta ; \bar{M}^{i}, R\right)$, and hence that $P^{i}(\Theta) \in$ $\mathcal{P}^{i}\left(\Theta^{i} ; \bar{M}^{-i}, R\right)$. Now, suppose that there exists another $P \in \mathcal{P}^{i}\left(\Theta ; \bar{M}^{-i}, R\right) \cap N^{i}(\bar{P})$. Then, for some $\tilde{\Theta}^{-i},\left(P, \tilde{\Theta}^{-i}\right) \in \mathcal{S}^{i}\left(\Theta ; \bar{M}^{-i}, R\right)$. Since each $\bar{M}^{j}$ gives no irrelevant price impact, it follows from Proposition 1 that $P$ allows no weak arbitrage opportunities, and then, from Lemma 2, that there exists $p \in \mathbb{R}^{S}$ such that $R^{\top} p=P$. By construction, $p=\left(R_{S}^{\top}\right)^{-1}\left(P_{1}, \ldots, P_{S}\right)^{\top} \in N^{i}(\bar{p})$ and

$$
\left(\left(R_{S}^{\top}\right)^{-1} P,\left(R \tilde{\Theta}^{j}\right)_{j \neq i}\right) \in \mathcal{S}^{i}\left(R \Theta ; \bar{m}^{-i}, \mathbb{I}_{S}\right) .
$$

Also by construction, $p \neq p^{i}(R \Theta)$ and $p \in N^{i}(\bar{p}) \cap \mathcal{P}^{i}\left(R \Theta ; \bar{m}^{-i}, \mathbb{I}_{S}\right)$, which is impossible. It follows that for each $i, P^{i}: N^{i}\left(\bar{\Theta}^{i}\right) \rightarrow N^{i}(\bar{P})$ is a (differentiable) local inverse demand: for all $\Theta \in N^{i}\left(\bar{\Theta}^{i}\right)$,

$$
\mathcal{P}^{i}\left(\Theta ; \bar{M}^{-i}, R\right) \cap N^{i}(\bar{P})=\left\{P^{i}(\Theta)\right\} ;
$$

since $\partial P^{i}\left(\bar{\Theta}^{i}\right)=\bar{M}^{i}$, it follows that $\bar{M}$ is mutually consistent.

For the second claim, suppose that $(\bar{P}, \bar{\Theta}, \bar{M})$ is an equilibrium for $\{u, e, R\}$. Market clearing is immediate: $\sum_{i} \bar{\theta}^{i}=\sum_{i} R \bar{\Theta}^{i}=R \sum_{i} \bar{\Theta}^{i}=0$. As before, we now show that each $\bar{\theta}^{i}$ is stable for $i$ given $\left(\bar{p}, \bar{m}^{i}\right)$, and that $\bar{m}$ is mutually consistent.

Suppose that $\bar{\theta}^{i}$ is not stable for $i$ given $\left(\bar{p}, \bar{m}^{i}\right)$. Then, we can fix $\theta$ such that

$$
-\left(\bar{p}+\bar{m}^{i}\left(\theta-\bar{\theta}^{i}\right)\right) \cdot \theta+u^{i}\left(e^{i}+\theta\right)>-\bar{p} \cdot \bar{\theta}^{i}+u^{i}\left(e^{i}+\bar{\theta}^{i}\right) .
$$

Fix any $\Theta$ such that $R \Theta=\theta$. Given that $(\bar{P}, \bar{\Theta}, \bar{M})$ and $(\bar{p}, \bar{\theta}, \bar{m})$ are $R$-associated, it follows by direct computation that

$$
-\left(\bar{P}+\bar{M}^{i}\left(\Theta-\bar{\Theta}^{i}\right)\right) \cdot \Theta+u^{i}\left(e^{i}+R \Theta\right)>-\bar{P} \cdot \bar{\Theta}^{i}+u^{i}\left(e^{i}+R \bar{\Theta}^{i}\right) .
$$


contradicting the fact that, in the economy with market $R, \bar{\Theta}^{i}$ is stable for $i$ given $\left(\bar{P}, \bar{M}^{i}\right)$.

Now, for each $i$, by mutual consistency of profile $\bar{M}$, there exist neighborhoods $N^{i}\left(\bar{\Theta}^{i}\right)$ and $N^{i}(\bar{P})$ and a differentiable local subequilibrium price function $P^{i}: N^{i}\left(\bar{\Theta}^{i}\right) \rightarrow N^{i}(\bar{P})$ such that $P^{i}\left(\bar{\Theta}^{i}\right)=\bar{P}$ and $\partial P^{i}\left(\bar{\Theta}^{i}\right)=\bar{M}^{i}$. Then, we can define open neighborhoods

$$
N^{i}\left(\bar{\theta}^{i}\right)=\left\{\theta \mid\left(\begin{array}{c}
R_{S}^{-1}\left(\theta-\bar{\theta}^{i}\right) \\
0
\end{array}\right)+\bar{\Theta}^{i} \in N^{i}\left(\bar{\Theta}^{i}\right)\right\}
$$

and

$$
N^{i}(\bar{p})=\left\{p \mid R^{\top} p \in N^{i}(\bar{P})\right\}
$$

By construction

$$
F(\theta)=\left(\begin{array}{c}
R_{S}^{-1}\left(\theta-\bar{\theta}^{i}\right) \\
0
\end{array}\right)+\bar{\Theta}^{i}
$$

maps $N^{i}\left(\bar{\theta}^{i}\right)$ into $N^{i}\left(\bar{\Theta}^{i}\right)$, and $G(P)=\left(R_{S}^{\top}\right)^{-1}\left(P_{1}, \ldots, P_{S}\right)^{\top}$ maps $N^{i}(\bar{P})$ into $N^{i}(\bar{p})$, so we can define $p^{i}=G \circ P^{i} \circ F: N^{i}\left(\bar{\theta}^{i}\right) \rightarrow N^{i}(\bar{p})$. Notice that $p^{i}\left(\bar{\theta}^{i}\right)=\left(R_{S}^{\top}\right)^{-1} P^{i}\left(\bar{\Theta}^{i}\right)=\bar{p}$, while

$$
\partial p^{i}\left(\bar{\theta}^{i}\right)=\left(\begin{array}{ll}
\left(R_{S}^{-1}\right)^{\top} & 0
\end{array}\right) \partial P^{i}\left(\bar{\Theta}^{i}\right)\left(\begin{array}{c}
R_{S}^{-1} \\
0
\end{array}\right)=\left(R_{S}^{-1}\right)^{\top} \bar{M}_{S}^{i} R_{S}^{-1}=\bar{m}^{i} .
$$

It only remains to show that for any $\theta \in N^{i}\left(\bar{\theta}^{i}\right), \mathcal{P}^{i}\left(\Theta ; \bar{M}^{i}, \mathbb{I}_{S}\right) \cap N^{i}(\bar{p})=\left\{p^{i}(\theta)\right\}$. Let $\Theta=$ $F(\theta)$. Notice that in the economy with market $R$, for some $\Theta^{-i},\left(P^{i}(\Theta), \Theta^{-i}\right) \in \mathcal{S}^{i}\left(\Theta ; \bar{M}^{-i}, R\right)$. Defining, for each $j \neq i, \theta^{j}=R \Theta^{j}$, it follows that $\left(p^{i}(\theta),\left(\theta^{j}\right)_{j \neq i}\right) \in \mathcal{S}^{i}\left(\theta ; \bar{m}^{i}, \mathbb{I}_{S}\right)$, and hence that $p^{i}(\theta) \in \mathcal{P}^{i}\left(\theta ; \bar{m}^{i}, \mathbb{I}_{S}\right)$. Now, suppose that there exists another $p \in \mathcal{P}^{i}\left(\theta ; \bar{m}^{i}, \mathbb{I}_{S}\right) \cap N^{i}(\bar{p})$. Then, for some $\tilde{\theta}^{-i},\left(p, \tilde{\theta}^{-i}\right) \in \mathcal{S}^{i}\left(\theta ; \bar{m}^{i}, \mathbb{I}_{S}\right)$. By construction, $P=R^{\top} p \in N^{i}(\bar{P})$ and $\left(R^{\top} p,\left(R_{S}^{-1} \tilde{\theta}^{j}, 0\right)_{j \neq i}\right) \in$ $\mathcal{S}^{i}\left(\left(R_{S}^{-1} \theta, 0\right) ; \bar{M}^{-i}, R\right)$. By Lemma 4 ,

$$
\mathcal{P}^{i}\left(\left(\begin{array}{c}
\left(R_{S}\right)^{-1} \theta \\
0
\end{array}\right) ; \bar{M}^{-i}, R\right)=\mathcal{P}^{i}\left(\Theta ; \bar{M}^{-i}, R\right),
$$

so $R^{\top} p \in \mathcal{P}^{i}\left(\Theta ; \bar{M}^{-i}, R\right)$. As before, by construction, $P \neq P^{i}(\Theta)$ and $P \in N^{i}(\bar{P}) \cap \mathcal{P}^{i}\left(\Theta ; \bar{M}^{-i}, R\right)$, which is impossible.

Q.E.D.

\section{Appendix A2: Existence and Generic Determinacy of Equilibria in Elementary SeCURities}

\section{EXISTENCE}

We now specialize the proof of Theorem 1 in [12], for the particular case treated here. All the arguments in this appendix are given for an economy with elementary securities, $\left\{u, e, \mathbb{I}_{S}\right\}$ satisfying our assumptions about preferences and endowments. Since we will only consider positive definite and diagonal price impact matrices, we identify these matrices with vectors in $\mathbb{R}_{++}^{S}$.

Let $h:\left(\mathbb{R}_{+}^{S}, \mathbb{R}_{++}^{S}\right)^{I-1} \rightarrow \mathbb{R}_{++}^{S}$ be the (component-wise) harmonic mean, divided by $(I-1)$; that is,

$$
h_{s}(m, v)=\left(\sum_{j}\left(m_{s}^{j}+v_{s}^{j}\right)^{-1}\right)^{-1} .
$$

Function $h$ is continuous, and it is well known that

$$
(I-1) h_{s}(m, v) \leq(I-1)^{-1} \sum_{j}\left(m_{s}^{j}+v_{s}^{j}\right),
$$


for all $m$, all $v$ and all $s$.

Claim 1. Let $\gamma_{u}$ be any strictly positive number, and let $(m, v) \in\left(\mathbb{R}_{+}^{S}, \mathbb{R}_{++}^{S}\right)^{I-1}$. Suppose that for some $s, m_{s}^{j} \leq(I-2)^{-1} \gamma_{u}$ and $v_{s}^{j} \leq \gamma_{u}$ for all $j$. Then, $h_{s}(m, v) \leq(I-2)^{-1} \gamma_{u}$.

Proof: This is immediate, since

$$
(I-1) h_{s}(m, v) \leq \frac{\sum_{j} m_{s}^{j}}{I-1}+\frac{\sum_{j} v_{s}^{j}}{I-1} \leq \frac{\gamma_{u}}{I-2}+\gamma_{u}
$$

Q.E.D.

Define, for each state $s, \mu_{s}=\max _{i}\left\{\partial u_{s}^{i}\left(e_{s}^{i}\right)\right\}$, a strictly positive real number. By the Inada conditions, we can fix, for each trader $i$ and state $s$, a revenue transfer $\hat{\theta}_{s}^{i}$ such that $\partial u_{s}^{i}\left(e_{s}^{i}+\hat{\theta}_{s}^{i}\right)=$ $\mu_{s}$. By concavity and Inada, $-e_{s}^{i}<\hat{\theta}_{s}^{i} \leq 0$.

Construct the (truncation) set of trades

$$
\mathcal{T}=\left\{\theta \in\left(\mathbb{R}^{S}\right)^{I}: \sum_{i} \theta_{s}^{i}=0 \text { and } \theta_{s}^{i} \geq \frac{1}{2}\left(\hat{\theta}_{s}^{i}-e_{s}^{i}\right) \text { for all } i \text { and all } s\right\}
$$

which is nonempty, convex and compact.

Define the function $V: \mathcal{T} \rightarrow\left(\mathbb{R}_{++}^{S}\right)^{I}$, componentwise, by letting $V_{s}^{i}(\theta)=-\partial^{2} u_{s}^{i}\left(e_{s}^{i}+\theta_{s}^{i}\right){ }^{26}$ Since $\mathcal{T}$ is compact, $V$ is continuous and $I$ and $S$ are finite, we can further define real numbers $\gamma_{u}=\max _{\theta \in \mathcal{T}} \max _{i, s} V_{s}^{i}(\theta)$ and $\gamma_{d}=\min _{\theta \in \mathcal{T}} \min _{i, s} V_{s}^{i}(\theta)$; these numbers, by construction, satisfy that $0<\gamma_{d} \leq \gamma_{u}$.

Define the function $\mathcal{U}:\left(\left[0,(I-2)^{-1} \gamma_{u}\right]^{S}\right)^{I} \rightarrow\left(\mathbb{R}^{S}\right)^{I}$ by

$$
\mathcal{U}(m)=\arg \max _{\theta \in \mathcal{T}} \sum_{i, s}\left(u_{s}^{i}\left(e_{s}^{i}+\theta_{s}^{i}\right)-\frac{1}{2} m_{s}^{i}\left(\theta_{s}^{i}\right)^{2}\right),
$$

which is well defined since $\mathcal{T}$ is compact and convex, each $u^{i}$ is continuous and strongly concave, and each $m_{s}^{i} \geq 0$. Define also the function $H:\left(\left[\gamma_{d}, \gamma_{u}\right]^{S}\right)^{I} \times\left(\left[0,(I-2)^{-1} \gamma_{u}\right]^{S}\right)^{I} \rightarrow\left(\mathbb{R}^{S}\right)^{I}$, by letting $H^{i}(m, v)=h\left(m^{-i}, v^{-i}\right)$. by

Finally, define the function $\mathcal{F}: \mathcal{T} \times\left(\left[\gamma_{d}, \gamma_{u}\right]^{S}\right)^{I},\left(\left[0,(I-2)^{-1} \gamma_{u}\right]^{S}\right)^{I} \rightarrow\left(\mathbb{R}^{S}\right)^{I} \times\left(\mathbb{R}^{S}\right)^{I} \times\left(\mathbb{R}^{S}\right)^{I}$,

$$
\mathcal{F}(\theta, v, m)=\left(\begin{array}{c}
\mathcal{U}(m) \\
V(\theta) \\
H(m, v)
\end{array}\right) .
$$

Claim 2. There exists $(\bar{\theta}, \bar{v}, \bar{m}) \in \mathcal{T} \times\left(\left[\gamma_{d}, \gamma_{u}\right]^{S}\right)^{I} \times\left(\left[0,(I-2)^{-1} \gamma_{u}\right]^{S}\right)^{I}$ such that $\mathcal{F}(\bar{\theta}, \bar{v}, \bar{m})=$ $(\bar{\theta}, \bar{v}, \bar{m})$.

Proof: By construction, $\mathcal{U}$ maps into $\mathcal{T}$, and $V$ into $\left(\left[\gamma_{d}, \gamma_{u}\right]^{S}\right)^{I}$. By Claim $1, H$ maps into $\left(\left[0,(I-2)^{-1} \gamma_{u}\right]^{S}\right)^{I}$. It follows that $\mathcal{F}$ maps a convex and compact set continuously into itself, so it has a fixed point.

Q.E.D.

Let a fixed point, $(\bar{\theta}, \bar{v}, \bar{m})$, of $\mathcal{F}$ be fixed.

ClaIm 3. For all $i$ and all $s$, it is true that $\bar{\theta}_{s}^{i}>\frac{1}{2}\left(\hat{\theta}_{s}^{i}-e_{s}^{i}\right)$.

26 This function is well defined, since, by construction, $\frac{\hat{\theta}_{s}^{i}-e_{s}^{i}}{2}>-e_{s}^{i}$, so $e_{s}^{i}+\theta_{s}^{i}>0$. 
Proof: Suppose not: suppose that for some $i$ and some $s, \bar{\theta}_{s}^{i}=\frac{1}{2}\left(\hat{\theta}_{s}^{i}-e_{s}^{i}\right)$. Since $\hat{\theta}_{s}^{i} \leq 0$, it follows that $\bar{\theta}_{s}^{i}<0$, which implies that, for some $j \neq i, \bar{\theta}_{s}^{j}>0$. By the definition of function $\mathcal{U}$, it follows, then, that

$$
\partial u_{s}^{i}\left(e_{s}^{i}+\bar{\theta}_{s}^{i}\right)-\bar{m}_{s}^{i} \bar{\theta}_{s}^{i} \leq \partial u_{s}^{j}\left(e_{s}^{j}+\bar{\theta}_{s}^{j}\right)-\bar{m}_{s}^{j} \bar{\theta}_{s}^{j},
$$

which is impossible, since

$$
\partial u_{s}^{i}\left(e_{s}^{i}+\bar{\theta}_{s}^{i}\right)-\bar{m}_{s}^{i} \bar{\theta}_{s}^{i}=\partial u_{s}^{i}\left(\frac{1}{2}\left(e_{s}^{i}+\hat{\theta}_{s}^{i}\right)\right)-\bar{m}_{s}^{i} \bar{\theta}_{s}^{i}>\mu_{s},
$$

whereas

$$
\partial u_{s}^{j}\left(e_{s}^{j}+\bar{\theta}_{s}^{j}\right)-\bar{m}_{s}^{j} \bar{\theta}_{s}^{j}<\partial u_{s}^{j}\left(e_{s}^{j}\right) \leq \mu_{s}
$$

Q.E.D.

We can now conclude that given preferences and endowments $\{u, e\}$, there exists an equilibrium in elementary securities $(\bar{p}, \bar{\theta}, \bar{m})$ satisfying that all price impact matrices are diagonal.

To see this, notice that, given concavity of all preferences and since $\bar{m}_{s}^{i} \geq 0$ for all $i$ and all $s$, it follows from Claim 3 that $\bar{\theta}$ actually solves program

$$
\max _{\theta: \sum_{i} \theta^{i}=0} \sum_{i, s}\left(u_{s}^{i}\left(e_{s}^{i}+\theta_{s}^{i}\right)-\frac{1}{2} \bar{m}_{s}^{i}\left(\theta_{s}^{i}\right)^{2}\right) .
$$

The first-order conditions of this problem immediately imply that there exist Lagrange multiplies $\bar{p} \in \mathbb{R}^{S}$ such that, for all $i$ and all $s$,

$$
\partial u_{s}^{i}\left(e_{s}^{i}+\bar{\theta}_{s}^{i}\right)-\bar{m}_{s}^{i} \bar{\theta}_{s}^{i}=\bar{p}_{s} .
$$

Identifying $\bar{m}^{i}$ and $\bar{v}^{i}$ with the diagonal, positive definite matrices

$$
\left(\begin{array}{ccc}
\bar{m}_{1}^{i} & \ldots & 0 \\
\vdots & \ddots & \vdots \\
0 & \ldots & \bar{m}_{S}^{i}
\end{array}\right) \text { and }\left(\begin{array}{ccc}
\bar{v}_{1}^{i} & \ldots & 0 \\
\vdots & \ddots & \vdots \\
0 & \ldots & \bar{v}_{S}^{i}
\end{array}\right)
$$

respectively, we get that:

1. $\sum_{i} \bar{\theta}^{i}=0$;

2. for each trader $i, \partial u^{i}\left(e^{i}+\bar{\theta}^{i}\right)-\bar{m}^{i} \bar{\theta}^{i}=\bar{p}$;

3. for each trader $i, \bar{m}^{i}=(I-1)^{-1} \mathcal{H}\left(\bar{m}^{-i}+\bar{v}^{-i}\right)$

4. for each trader $i, \bar{v}^{i}=-\partial^{2} u^{i}\left(e^{i}+\bar{\theta}^{i}\right)$.

Then, it is immediate from Lemma 1 that $(\bar{p}, \bar{\theta}, \bar{m})$ is an equilibrium.

\section{Generic Determinacy}

Assume now that, moreover, each Bernoulli function $u_{s}^{i}$ is of class $\mathbb{C}^{3}$ and satisfies that $\partial^{2} u_{s}^{i}$ is bounded strictly below zero.

Denote by $\mathbb{U}$ the class of Bernoulli functions that satisfy all the assumptions we have imposed, ${ }^{27}$ and endow this set with the topology of $\mathbb{C}^{3}$ uniform convergence on compacta. ${ }^{28}$ The

27 Namely, that each $u_{s}^{i}$ is differentiably strictly monotonic and differentiably strictly concave, and satisfies Inada conditions. Unlike in Proposition 5, we do not require now that these functions be CRRA. 28 See [1]. 
space of economies we now consider is the set $\left(\mathbb{R}_{++}^{S}\right)^{I} \times\left(\mathbb{U}^{S}\right)^{I}$, endowed with the product topology.

We want to show that, in a dense subset of these economies, all equilibria in elementary securities are locally unique.

To do this, fix an economy $\{\bar{u}, \bar{e}\}$ and an open neighborhood $\mathcal{O}$ of that economy. We need to show that for at least one economy $\{u, e\}$ in $\mathcal{O}$ the property of local uniqueness holds true.

\section{A Finite-Dimensional Subspace of Economies}

As the space of economies is an infinite-dimensional manifold, it is convenient to do our analysis in a finite-dimensional, local subspace of economies. The strategy is to show that in this subspace we can find the economy $\{u, e\}$, where local uniqueness holds, arbitrarily close to $\{\bar{u}, \bar{e}\}$. In this section, we define the subspace of economies.

Fix $\epsilon>0$, and a $\mathbb{C}^{\infty}$ function $\rho: \mathbb{R}_{++} \rightarrow \mathbb{R}_{+}$such that

$$
\rho(x)= \begin{cases}1, & \text { if } x<\max _{s}\left\{\sum_{i} \bar{e}_{s}^{i}\right\}+I \epsilon+1 \\ 0, & \text { if } x \geq \max _{s}\left\{\sum_{i} \bar{e}_{s}^{i}\right\}+I \epsilon+2\end{cases}
$$

Also, define for each $i$ and $s$ the "perturbed Bernoulli" function $u_{s}^{i}: \mathbb{R}_{++} \times \mathbb{R} \rightarrow \mathbb{R}$ by

$$
u_{s}^{i}\left(x_{s}^{i}, \delta_{s}^{i}\right)=\bar{u}_{s}^{i}\left(x_{s}^{i}\right)+\frac{1}{2} \rho\left(x_{s}^{i}\right) \delta_{s}^{i}\left(x_{s}^{i}\right)^{2} .
$$

Since each $\max _{s}\left\{\sum_{i} \bar{e}_{s}^{i}\right\}$ is finite, there exists $\bar{\delta}>0$ such that for all $-\bar{\delta}<\delta_{s}^{i}<\bar{\delta}$, function $u_{s}^{i}\left(\cdot, \delta_{s}^{i}\right) \in \mathbb{U}$

Now, we consider the finite dimensional subset of economies defined by endowments $e \in$ $B_{\epsilon}(\bar{e})$, and preferences given, for each $i$ and $s$, by $u_{s}^{i}\left(\cdot, \delta_{s}^{i}\right)$ for some $\delta_{s}^{i} \in B_{\bar{\delta}}(0)$. This is a submanifold parameterized by $B_{\epsilon}(\bar{e}) \times B_{\bar{\delta}}(0)^{S I}$. For notational simplicity, we identify the vector $\delta^{i}=\left(\delta_{s}^{i}\right)_{s}$ with the diagonal matrix $\operatorname{diag}\left(\delta_{s}^{i}\right)$, and denote $\delta=\left(\delta^{i}\right)_{i=1}^{I}$. Similarly, we again identify the vector $m^{i}=\left(m_{s}^{i}\right)_{s}$ with the diagonal matrix $\operatorname{diag}\left(m_{s}^{i}\right)$.

\section{TRANSVERSALITY}

Now, consider the function $\mathcal{G}: \mathbb{R}^{S} \times\left(\mathbb{R}^{S}\right)^{I} \times\left(\mathbb{R}_{++}^{S}\right)^{I} \times B_{\epsilon}(\bar{e}) \times B_{\bar{\delta}}(0) \rightarrow\left(\mathbb{R}^{S}\right)^{I} \times \mathbb{R}^{S} \times\left(\mathbb{R}^{S}\right)^{I}$, defined by

$$
\mathcal{G}(p, \theta, m, e, \delta)=\left(\begin{array}{c}
\vdots \\
p+m^{i} \theta^{i}-\partial_{x} u^{i}\left(e^{i}+\theta^{i}, \delta^{i}\right) \\
\vdots \\
\sum_{i} \theta^{i} \\
\vdots \\
\left(m_{s}^{i} \sum_{j \neq i}\left(m_{s}^{j}-\partial_{x, x}^{2} u_{s}^{j}\left(e_{s}^{j}+\theta_{s}^{j}, \delta_{s}^{j}\right)^{-1}\right)-1\right)_{s} \\
\vdots
\end{array}\right) .
$$

It follows from lemma 1 that $(p, \theta, m)$ is an equilibrium in elementary securities for economy $(e, \delta)$ if, and only if, $\mathcal{G}(p, \theta, m, e, \delta)=0$.

Claim 4. Function $\mathcal{G}$ is transverse to 0 : whenever $\mathcal{G}(p, \theta, m, e, \delta)=0$, matrix $\partial \mathcal{G}(p, \theta, m, e, \delta)$ has full (row) rank. 
Proof: Let $(p, \theta, m, e, \delta)$ be such that $\mathcal{G}(p, \theta, m, e, \delta)=0$. Since each $\theta_{s}^{i}>-e_{s}^{i}$ and $\sum_{i} \theta^{i}=0$, it follows that each $\theta_{s}^{i} \leq \sum_{j} e_{s}^{i}<\sum_{j} \bar{e}_{s}^{j}+I \epsilon$, and then, by construction, it follows that for any $x$ in a neighborhood of $e^{i}+\theta^{i}$,

$$
u^{i}\left(x, \delta^{i}\right)=\bar{u}^{i}(x)+\frac{1}{2} x^{\top} \delta^{i} x .
$$

By direct computation, and considering only the derivatives with respect to $\left(\theta^{1}, \ldots, \theta^{I}, e^{1}\right)$ and $\left(m^{1}, \ldots, m^{I}, \delta^{1}, \ldots, \delta^{I}\right)$, matrix $\partial \mathcal{G}(p, \theta, m, e, \delta)$ can be written in partition form as

$$
\Gamma=\left(\begin{array}{ll}
\Gamma_{1,1} & \Gamma_{1,2} \\
\Gamma_{2,1} & \Gamma_{2,2}
\end{array}\right)
$$

where $^{29} \Gamma_{2,2}=\left(\begin{array}{cc}\Gamma_{2,2}^{L} & \Gamma_{2,2}^{R}\end{array}\right)$,

$$
\begin{aligned}
& \Gamma_{1,1}=\left(\begin{array}{cccc}
m^{1}-\partial^{2} \bar{u}^{1}-\delta^{1} & \ldots & 0 & -\partial^{2} \bar{u}^{1}-\delta^{1} \\
\vdots & \ddots & \vdots & \vdots \\
0 & \ldots & m^{I}-\partial^{2} \bar{u}^{I}-\delta^{I} & 0 \\
\mathbb{I} & \ldots & \mathbb{I} & 0
\end{array}\right), \\
& \Gamma_{1,2}=\left(\begin{array}{cccccc}
\operatorname{diag}\left(\theta_{s}^{1}\right) & \ldots & 0 & -\operatorname{diag}\left(\theta_{s}^{1}\right) & \ldots & 0 \\
\vdots & \ddots & \vdots & \vdots & \ddots & \vdots \\
0 & \ldots & \operatorname{diag}\left(\theta_{s}^{I}\right) & 0 & \ldots & -\operatorname{diag}\left(\theta_{s}^{I}\right) \\
0 & \ldots & 0 & 0 & \ldots & 0
\end{array}\right) \text {, } \\
& \Gamma_{2,2}^{L}=\left(\begin{array}{ccc}
\sum_{j \neq 1}\left(m^{j}-\partial^{2} \bar{u}^{j}-\delta^{j}\right)^{-1} & \ldots & -\operatorname{diag}\left(\frac{m_{s}^{1}}{\left(m_{s}^{I}-\partial^{2} \bar{u}_{s}^{I}-\delta_{s}^{I}\right)^{2}}\right) \\
\vdots & \ddots & \vdots \\
-\operatorname{diag}\left(\frac{m_{s}^{I}}{\left(m_{s}^{1}-\partial^{2} \bar{u}_{s}^{1}-\delta_{s}^{1}\right)^{2}}\right) & \ldots & \sum_{j \neq I}\left(m^{j}-\partial^{2} \bar{u}^{j}-\delta^{j}\right)^{-1}
\end{array}\right)
\end{aligned}
$$

and

$$
\Gamma_{2,2}^{R}=\left(\begin{array}{ccc}
0 & \ldots & \operatorname{diag}\left(\frac{m_{s}^{1}}{\left(m_{s}^{I}-\partial^{2} \bar{u}_{s}^{I}-\delta_{s}^{I}\right)^{2}}\right) \\
\vdots & \ddots & \vdots \\
\operatorname{diag}\left(\frac{m_{s}^{I}}{\left(m_{s}^{1}-\partial^{2} \bar{u}_{s}^{1}-\delta_{s}^{1}\right)^{2}}\right) & \ldots & 0
\end{array}\right)
$$

Notice that matrix $\Gamma_{1,1}$ is nonsingular, by standard arguments, given our assumptions on concavity of preferences and the fact that each price impact matrix is positive definite. ${ }^{30}$ Then, to see that $\Gamma$ has full row rank, it suffices to observe that there exists an $(S I+S+S I+S I) \times S I$ matrix $A$ such that

$$
\Gamma A=\left(\begin{array}{l}
0 \\
\mathbb{I}
\end{array}\right)
$$

29 The precise form of matrix $\Gamma_{2,1}$ is immaterial for computations below.

30 To see this, the argument is the one that gives determinacy in competitive models: (i) notice that the submatrix composed by the first $I$ superrows and $I$ supercolumns is nonsingular, since it is a diagonal matrix of positive numbers: each $m_{s}^{i}>0$ and each $\partial^{2} \bar{u}_{s}^{i}\left(e_{s}^{i}+\theta_{s}^{i}\right)+\delta_{s}^{i}<0$, as $u_{s}^{i}\left(\cdot, \delta_{s}^{i}\right)$ is strongly concave; (ii) Now, to see that the whole $\Gamma_{1,1}$ is nonsingular, it suffices to observe that if one constructs the $S(I+1) \times S$ matrix $A$ by letting the first $S \times S$ superrow be $\mathbb{I}$, letting the last $S \times S$ superrow be $-\left(\partial^{2} \bar{u}^{1}\left(e^{i}+\theta^{i}\right)+\delta^{1}\right)^{-1}\left(m^{1}-\partial^{2} \bar{u}^{1}-\delta^{1}\right)$, and letting every other element be 0 , we get, by direct computation, that

$$
\Gamma_{1,1} A=\left(\begin{array}{l}
0 \\
\mathbb{I}
\end{array}\right) .
$$


For this, just let matrix

$$
A=\left(\begin{array}{c}
0 \\
A_{2}
\end{array}\right)
$$

where

$$
A_{2}=\left(\begin{array}{ccc}
m^{1} & \ldots & 0 \\
\vdots & \ddots & \vdots \\
0 & \ldots & m^{I} \\
m^{1} & \ldots & 0 \\
\vdots & \ddots & \vdots \\
0 & \ldots & m^{I}
\end{array}\right)
$$

By direct computation, the result follows from the fact that, for each $i$,

$$
m^{i} \sum_{j \neq i}\left(m^{j}-\partial^{2} \bar{u}^{j}\left(e^{j}+\theta^{j}\right)-\delta^{j}\right)^{-1}=\mathbb{I}
$$

Q.E.D.

Now, we argue that for almost all economies in the finite-dimensional subspace of economies all equilibria are locally isolated.

Claim 5. For a full-measure subset of $B_{\epsilon}(\bar{e}) \times B_{\bar{\delta}}(0)^{S I}$, it is true that for any equilibrium in elementary securities $(\bar{p}, \bar{\theta}, \bar{m})$ there exists an open neighborhood in which there are no more equilibria for the same economy.

Proof: By Claim 4 and the Transversality Theorem (see [4]), it follows that for any $(e, \delta)$ in a full measure subset of $B_{\epsilon}(\bar{e}) \times B_{\bar{\delta}}(0)^{S I}$, function $\mathcal{G}(\cdot, e, \delta)$ is transverse to 0 . If $(\bar{p}, \bar{\theta}, \bar{m})$ is an equilibrium for $(e, \delta)$, it must be that $\mathcal{G}(\bar{p}, \bar{\theta}, \bar{m}, e, \delta)=0$, and hence that $\partial_{p, \theta, m} \mathcal{G}(\bar{p}, \bar{\theta}, \bar{m}, e, \delta)$ has full row rank. But $\partial_{p, \theta, m} \mathcal{G}$ is a square matrix, so $\partial_{p, \theta, m} \mathcal{G}(\bar{p}, \bar{\theta}, \bar{m}, e, \delta)$ is nonsingular and hence, by the inverse function theorem, $\mathcal{G}(\cdot, e, \delta)$ is a bijection in a neighborhood of $(\bar{p}, \bar{\theta}, \bar{m})$. Q.E.D.

\section{DENSENESS}

The last claim shows that in almost every economy in the finite-dimensional subspace, the property of local uniqueness holds. To complete our argument that the set of economies where the property holds is dense in the general space of economies, it suffices to show that the finite subspace of economies intersects the pre-fixed neighborhood $\mathcal{O}$ of $\{\bar{u}, \bar{e}\}$. But this is true, because the topology with which the space of economies is endowed is metrizable (see [11, p. 428]), and in this metric the distance from $\bar{u}_{s}^{i}=u_{s}^{i}(\cdot, 0)$ to $u_{s}^{i}\left(\cdot, \delta_{s}^{i}\right)$ can be made arbitrarily small by choosing a small enough $\delta_{s}^{i}>0$. 


\section{References}

[1] Aliprantis, C. and Border, K., Infinite Dimensional Analysis, Springer, 1999.

[2] Chan, K.C. and Lakonishok, J., "The behavior of stock price around institutional trades," Journal of Finance 50, 1147-1174, 1995.

[3] Gompers, P. and Metrick, A., "Institutional investors and equity prices," Quarterly Journal of Economics 116, 1, 229-259, 2001.

[4] Guillemin, V. and A. Pollack, Differential Topology, Prentice Hall, 1974.

[5] Hart, O. "Imperfect competition in general equilibrium: an overview of recent work," in Arrow K. and Honkapohja S. (Eds.) Frontiers of Economics, Blackwell, 1985.

[6] Keim, D. and Madhavan, A., "The upstairs market for large-block transactions: analysis and measurement of price effects," Review of Financial Studies 9, 1-39, 1996.

[7] Kyle, A. "Informed Speculation and Imperfect Competition" Review of Economic Studies $56,317-356,1989$.

[8] Negishi, T. "Monopolistic competition and general equilibrium," Review of Economic Studies 28, 196-201, 1961.

[9] Perold, A. "The implementation shortfall: papers versus reality," Journal of Portfolio Management 14, 3, 1988.

[10] Vayanos, D. "Strategic Trading and Welfare in a Dynamic Market" Review of Economic Studies 66, 219-254, 1999.

[11] Villanacci, A., L. Carosi, P. Benevieri and A. Battinelli, Differential Topology and General Equilibrium with Complete and Incomplete Markets, Kluwer, 2002.

[12] Weretka, M. "Endogenous Market Power," Mimeo, 2005, available from http://www.ssc.wisc.edu/ mweretka/Research.

[13] Weretka, M. "Discovery of Market Power in Bilateral Oligopoly," Mimeo, 2007, available from http://www.ssc.wisc.edu/ mweretka/Research.

[14] Weretka, M. "Cross-Market Effects in Thin Markets," Mimeo, 2007, available from http://www.ssc.wisc.edu/ mweretka/Research.

[15] Weretka, M. "Asset Valuation in Thin Financial Markets," Mimeo, 2007, available from http://www.ssc.wisc.edu/ mweretka/Research.

[16] Rostek, M. and Weretka, M. "Term structure of Price Impact," Mimeo, 2007, available from http://www.ssc.wisc.edu/ mweretka/Research. 